\title{
Neural field model of memory-guided search
}

\author{
Zachary P. Kilpatrick ${ }^{1,2, *}$ and Daniel B. Poll ${ }^{3,4}$ \\ ${ }^{1}$ Department of Applied Mathematics, University of Colorado, Boulder, Colorado 80309, USA \\ ${ }^{2}$ Department of Physiology and Biophysics, University of Colorado School of Medicine, Aurora, Colorado 80045, USA \\ ${ }^{3}$ Department of Mathematics, University of Houston, Houston, Texas 77204, USA \\ ${ }^{4}$ Department of Engineering Sciences and Applied Mathematics, Northwestern University, Evanston, Illinois 60208, USA
}

(Received 6 September 2017; revised manuscript received 30 October 2017; published 18 December 2017)

\begin{abstract}
Many organisms can remember locations they have previously visited during a search. Visual search experiments have shown exploration is guided away from these locations, reducing redundancies in the search path before finding a hidden target. We develop and analyze a two-layer neural field model that encodes positional information during a search task. A position-encoding layer sustains a bump attractor corresponding to the searching agent's current location, and search is modeled by velocity input that propagates the bump. A memory layer sustains persistent activity bounded by a wave front, whose edges expand in response to excitatory input from the position layer. Search can then be biased in response to remembered locations, influencing velocity inputs to the position layer. Asymptotic techniques are used to reduce the dynamics of our model to a low-dimensional system of equations that track the bump position and front boundary. Performance is compared for different target-finding tasks.
\end{abstract}

DOI: 10.1103/PhysRevE.96.062411

\section{INTRODUCTION}

Most motile organisms rely on their ability to search [1]. The processes of identifying habitats, food sources, mates, and predators make use of visual search combined with spatial navigation $[2,3]$. One guiding principle used to evaluate how organisms implement search is the exploration-exploitation tradeoff [4,5]. Exploiting one's current position to search locally has a low cost, whereas exploring by moving to another search position has a higher cost but a potentially higher reward [6]. Many organisms have developed search strategies that attempt to manage this tradeoff in a robust way $[7,8]$. Mathematical models of search can quantify the resources expended and yielded by different strategies, showing how managing the explore-exploit tradeoff is key $[9,10]$.

Memoryless stochastic processes are commonly used to model the dynamics of searching organisms [11]. Such models prescribe equations for an agent that moves according to pure diffusion [12], mixed advection-diffusion [13,14], or even local diffusion punctuated by large deviations in position [15]. None of these search strategies relies on information about previous locations the agent has visited. However, some studies, particularly studies that focus on visual search, have examined the impact of memory on random search processes [16]. There is evidence suggesting organisms tend to guide their gaze away from locations they have already examined $[17,18]$, a mechanism often called inhibition-of-return (IOR). While the degree to which IOR facilitates exploration continues to be debated [19-21], recent studies suggest return saccades to previously visited locations are less frequent than they would be for a memoryless search process [22].

Neural mechanisms underlying memory of previous visual targets are relatively unknown, but function magnetic resonance imaging (fMRI) studies in humans have shed light on specific brain areas that might be involved [23]. One

\footnotetext{
*zpkilpat@colorado.edu; daniel.poll@ northwestern.edu
}

candidate region is the superior colliculus (SC), known to be involved in oculomotor planning [24]. Unilateral lesioning of SC disables IOR during visual search performed in the visual hemifield linked to the damaged half of the brain [25]. Other oculomotor programming areas linked to IOR include the supplementary eye field (SEF) and frontal eye field (FEF) $[26,27]$. Detectable activation of these regions suggests models of IOR should represent previous search locations using some form of persistent neural activity. In this way, there is a parallel between inhibitory tagging of previous locations and working memory, which also engages persistent activity for its implementation [28,29]. However, it remains unclear how persistent activity might be initiated and utilized by a neural circuit to successfully implement search guided by IOR.

There is also evidence that top-down signals guided by biased competition can drive attention in a preferred direction based on a decision-making process that selects relevant places in a scene to look [34,35]. Visual search guidance can be thought of as a spatially directed attention process, shaped by neural activity in ventral stream areas [36,37]. These regions are known to possess a topographic organization via individual neurons' receptive fields. For instance, both V4 [38] and SC [39] are known to possess a retinotopic salience map that guides visual search direction, so such areas are likely involved in the oculomotor planning process. There are changes in the neural activity in localized regions of V4 associated with saccades to a particular part of the visual search area [40]. This suggests that there are likely multiple areas involved in visual search planning and feedback, but in this initial study we focus on a simple model that collapses the neural underpinnings of search planning to a single area. There has been extensive experimental work showing that propagating patterns of neural activity are relevant to visual processing [41-43], so it is reasonable to expect that the brain might exploit such frameworks to guide visual search.

We develop a model of memory-guided search that stores an agent's present search location as well as previously visited search locations. Our model consists of two neural field 
(a)

It is, of course, an indispensable part of a scrivener's business to verify the accuracy of his copy, word by word. Where there are two or more scriveners in an office, they assist each other in this examination, one reading from the copy, the other holding the original. It is a very dull, wearisome, and lethargic affair. I can readily imagine that to some sanguine temperaments it would be altogether intolerable.

(b)

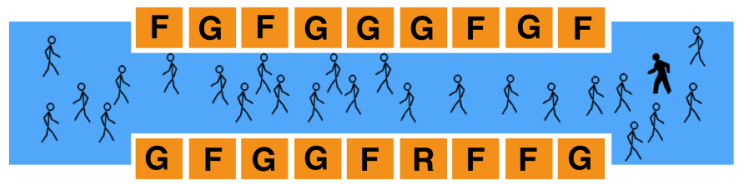

(e)

(d)
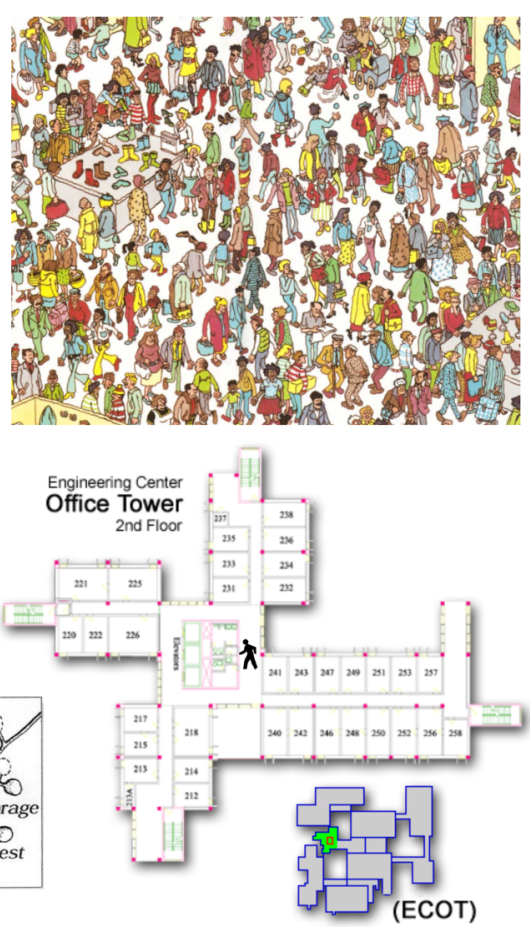

(c)
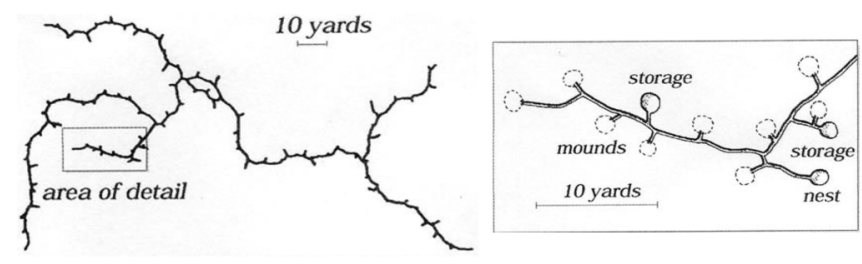

(ECOT)

FIG. 1. Examples of search tasks. Effectively one-dimensional: (a) Find the number in the above quote from Bartleby the Scrivener by Herman Melville (1853) [30]. (b) Bolded agent must find the restroom (R) among the food vendors (F) and gates (G) in a narrow airport terminal. (c) Gopher must find its nest within a narrow network of underground tunnels [31]. Multidimensional: (d) Find Waldo among a crowd of non-Waldo visual distractors [32]. (e) Student looking for their professor's office in the multifloor (three-dimensional) Engineering Center Office Tower at University of Colorado Boulder [33].

equations: One neural field layer captures the position of the searcher and the other describes the memory of visited locations. To systematically analyze our neural field model, we focus on one-dimensional search tasks such as scanning lines of text for a specific word [Fig. 1(a)]; searching a long corridor [Fig. 1(b)]; or foraging in a tunnel system [Fig. 1(c)]. We idealize these examples by considering the problem of searching along a one-dimensional segment or a radial arm maze. After our analysis of the one-dimensional system, we discuss how our model can be extended to two-dimensional search tasks, such as finding an object in a picture [Fig. 1(d)] or higher dimensions [Fig. 1(e)].

We proceed by presenting our one-dimensional model of memory of visited spatial locations in Sec. II. This neural field model suggests a basic neural circuit architecture for encoding previously searched regions of a spatial domain. Subsequently, we analyze the existence and stability of stationary solutions to our model equations in Sec. III. The position of the searching agent is represented by a stationary pulse (bump) solution in the absence of velocity inputs, and the previously searched region is bounded by two stationary front interfaces. Our analysis provides us with intuition as to how model parameters shape the spatial resolution and robustness of the memory representation. After this, we carry out a low-dimensional reduction of our model, so that we can capture its dynamics by tracking the location of the bump and front interfaces (Sec. IV). In fact, this captures the dynamics of the full neural field quite well. Subsequently, we evaluate the performance of memory-guided search, when the velocity inputs are shaped by the previously visited locations stored in memory (Sec. V). Memory-guided search does not improve the speed of searches along a single segment but does improve search across multiple connected segments, as in a radial arm maze. Lastly, we discuss extensions to planar domains in Sec. VI.

\section{MULTILAYER NEURAL FIELD MODEL}

Our model of memory-guided search is composed of two layers of stimulus-tuned neurons corresponding to locations of the searching agent. This is mostly motivated by studies of visual search [20], but there could be IOR mechanisms that shape the search processes driven by organisms' idiothetic ${ }^{1}$ navigation [1]. The first layer of the network encodes the agent's position [Fig. 2(a)], and is driven by velocity input, which the network integrates (Sec. IV). This layer projects to the second layer, which encodes a memory of locations the agent has visited [Fig. 2(b)]. Closed-loop control of the velocity input can be implemented using the memory and position layer to determine the agent's next search location. We discuss this in more detail in Sec. V.

We employ a neural field model describing the population activity of two layers: a position-encoding layer $u(x, t)$, which

\footnotetext{
${ }^{1}$ Idiothetic literally means "self-proposition" (from Greek) and describes the use of self-motion cues such as vestibular, optic flow, or proprioception. Spatial navigation can be performed using purely self-motion cues via path integration.
} 
(a)
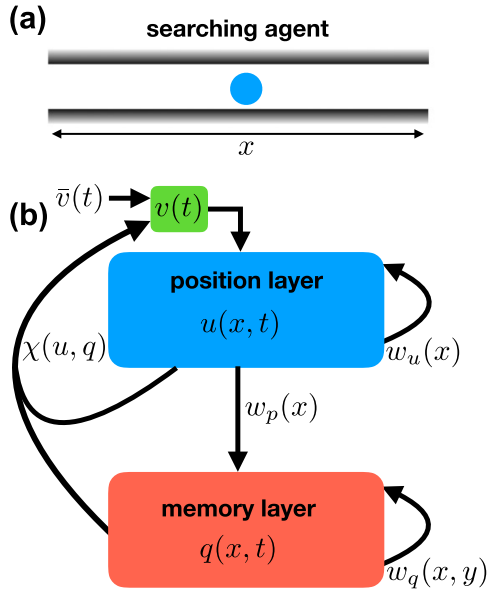
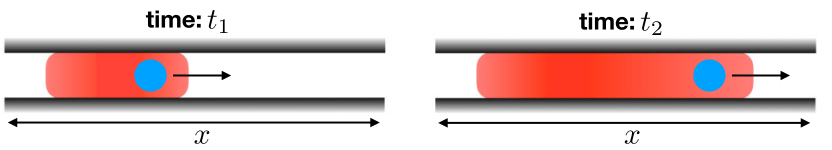

(c)
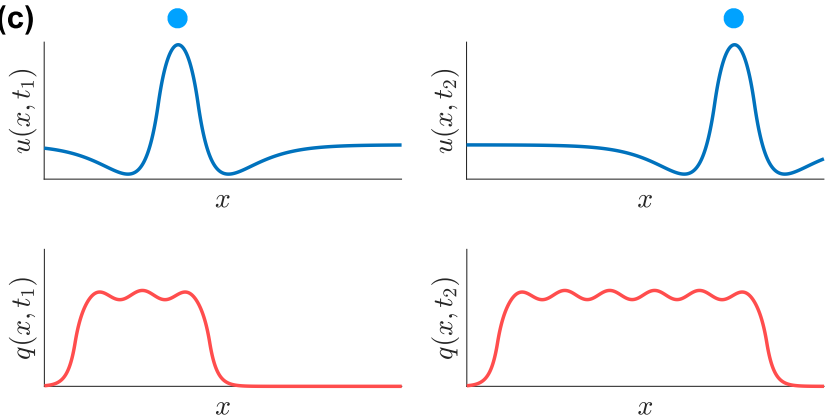

FIG. 2. Multilayer neural field model describes neural computations involved in memory-guided search. (a) Searching agent (blue dot) moves along a one-dimensional domain, storing a memory of its previously visited locations (red regions), expanding with time. (b) Multilayer neural field model contains a position-encoding layer $[u(x, t)]$ and memory-encoding layer $[q(x, t)]$, representing previously visited locations. Position-encoding layer $u(x, t)$ receives a velocity input $v(t)$, which is the searching agent's true velocity, the product of a baseline search velocity $\bar{v}(t)$ and a control signal $\chi(u, q)$ based on the memory of previously visited locations. Recurrent connections $w_{u}(x)$ within layer $u(x, t)$ sustain persistent bump of activity representing location, and this location information is relayed via feedforward connections $w_{p}(x)$ to layer $q(x, t)$. A front of activity is sustained in $q(x, t)$ via recurrent connections $w_{q}(x, y)$. (c) Peak of bump in position layer $[u(x, t)]$ represents agent's current position. Input to memory layer activates neural population $[q(x, t)]$ that represent previously visited locations.

supports a bump attractor solution, and a memory layer $q(x, t)$, which supports stationary front solutions [Fig. 2(b)],

$$
\begin{gathered}
u_{t}=-u+w_{u} * H\left(u-\theta_{u}\right)-v(t)\left(w_{u}^{\prime}\right) * H\left(u-\theta_{u}\right), \\
q_{t}=-q+w_{q} \bar{*} H\left(q-\theta_{q}\right)+w_{p} * H\left(u-\theta_{u}\right) .
\end{gathered}
$$

The neural fields $u(x, t)$ and $q(x, t)$ represent the normalized synaptic input to the position-encoding and memory layers over $x \in(-\infty, \infty)$. Recurrent coupling in the position layer is described by the convolution $w_{u} * H\left(u-\theta_{u}\right)=\int_{-\infty}^{\infty} w_{u}(x-$ $y) H\left(u(y, t)-\theta_{u}\right) d y$ with weight function $w_{u}(x)$, whereas velocity-modulation of coupling occurs through the convolution $w_{u}^{\prime} * H\left(u-\theta_{u}\right)=\int_{-\infty}^{\infty} w_{u}^{\prime}(x-y) H\left(u(y, t)-\theta_{u}\right) d y$. We model the total velocity input $v(t)$ as a product of a baseline search velocity, $\bar{v}(t)$, and a control term $\chi(u, q)$ that describes changes in velocity due to remembered locations. Thus, the function $\chi(u, q)$ incorporates the memory of the current position $u(x, t)$ and previously visited locations $q(x, t)$. Further details on our choice of $\chi(u, q)$ are given in Appendix D. The memory layer $q(x, t)$ receives feedforward input via a convolution involving the weight function $w_{p}(x)$, and its recurrent coupling is spatially heterogeneous, so $w_{q} \bar{*} H\left(q-\theta_{q}\right)=$ $\int_{-\infty}^{\infty} w_{q}(x, y) H\left(q(y, t)-\theta_{q}\right) d y$. Each layer $j$ has a Heaviside firing rate function, $H\left(u-\theta_{j}\right)=\left\{1: u \geqslant \theta_{j} ; 0: u<\theta_{j}\right\}$, with its own constant threshold parameter $\theta_{u}$ and $\theta_{q}$. We discuss the model's constituent functions in more detail below.

\section{A. Position-encoding layer}

Both the direction of visual gaze [44] and an animal's idiothetic location [45] are known to be encoded by position-tuned cells $[46,47]$. Network ensembles with excitatory coupling between cells with similarly tuned stimulus preference and effective inhibition between cells with dissimilar stimulus preference generate localized packets of activity representing location $[48,49]$. Together with well-accepted models of velocity input to networks with positional memory [50], this suggests a locally excitatory $\left(w_{u}>0:|x-y|<r\right)$ and distally inhibitory $\left(w_{u}<0:|x-y|>r\right)$ weight kernel for the recurrent connectivity of the position layer $u(x, t)$, Eq. (1). We consider the "wizard hat" [51,52], for explicit calculations:

$$
w_{u}(x)=(1-|x|) e^{-|x|},
$$

so $w_{u}(x)>0$ when $|x|<1$ and $w_{u}(x)<0$ when $|x|>1$. The Heaviside step nonlinearity in Eq. (1), $H\left(u-\theta_{u}\right)$, converts synaptic input to output firing rate [53]. More nuanced firing rate functions are possible but complicate calculations and do not substantially alter the qualitative results [54]. Velocity inputs are modeled by the final term in Eq. (1), which propagates stationary bump solutions in the direction of the time-dependent velocity $v(t)$ [50]. This feature is ensured by the form of the weight function $w_{u}^{\prime}(x)$ in the convolution, which results in a translation of the bumps, as we demonstrate in Sec. IV. Given the weight function Eq. (3), we have

$$
w_{u}^{\prime}(x)=-\operatorname{sgn}(x)(2-|x|) e^{-|x|},
$$

where $\operatorname{sgn}(x)= \pm 1$ if $x \gtrless 0$ and $\operatorname{sgn}(0)=0$, so a jump discontinuity arises at $x=0$, which is mollified by the integration in Eq. (1).

\section{B. Memory layer}

As we have discussed, IOR biases organisms' search strategy, so they are less likely to visit locations they have already scanned [18,55]. We account for neural hardware capable of storing visited locations over appreciable periods of time (e.g., at least several seconds [20]). Note, the weight kernel $w_{q}(x, y)$ in Eq. (2) is spatially heterogeneous, and the scale of the heterogeneity may be set by environmental 
landmarks [56] or the spatial extent of receptive fields or hypercolumns in the visual cortex [57]. Heterogeneity can stabilize activity, pinning wavefronts to a stationary location [58-60]. We assume spatial heterogeneity is periodic, given by a cosine-modulated exponential:

$$
\begin{aligned}
w_{q}(x, y) & :=\left[1+\sigma w_{h}(y)\right] \bar{w}_{q}(x-y) \\
& =[1+\sigma \cos (n y)] \frac{e^{-|x-y|}}{2},
\end{aligned}
$$

so the weight function is a homogeneous kernel $\bar{w}_{q}(x-y)$ modulated by a periodic heterogeneity $w_{h}(y)$. Varying the amplitude $\sigma$ increases the parameter range for which front solutions are pinned. For perturbative results, we assume $0<\sigma \ll 1$. The parameter $n$ sets the number of stable conformations of wavefronts over the length $2 \pi$. Input from the position layer is weak, as compared with local recurrent connections, and excitatory,

$$
w_{p}(x)=I_{0} \frac{\alpha \mathrm{e}^{-\alpha|x|}}{2},
$$

so typically we assume $0<I_{0} \ll 1$. Varying the inverse spatial scale $\alpha$ shapes the precision with which position information is sent from the position layer to the memory layer. Changing $\alpha$ has minimal impact on our qualitative results, so we set $\alpha=1$.

As mentioned, velocity $v(t)$ is a nonlinear combination of a baseline velocity $\bar{v}(t)$, which would guide the agent with no memory-based feedback, and a control function $\chi(u, q)$, which depends on both the position $u(x, t)$ and the memory $q(x, t)$ layer activities. We discuss effects of this control in Sec. V, where we examine performance of the model in two different types of search tasks.

\section{STATIONARY SOLUTIONS}

We begin by studying the existence and stability of stationary solutions of the neural field model, Eq. (1), (2), in the case of no velocity input $(v(t) \equiv 0)$ and strong input position input and heterogeneity ( $I_{0}$ and $\sigma$ arbitrary). Understanding these stationary solutions, and the effect of the position layer on the memory layer, provides us with insight needed to project the neural field to a low-dimensional model that can be more easily analyzed. Subsequently, we consider the effect of closed-loop control on the velocity input based upon the combination of position and memory layer activities.

\section{A. Existence}

We begin with the stationary equations associated with Eqs. (1) and (2), finding

$$
\begin{aligned}
& U(x)=w_{u} * H\left(U(x)-\theta_{u}\right), \\
& Q(x)=w_{q} \bar{*} H\left(Q(x)-\theta_{q}\right)+w_{p} * H\left(U(x)-\theta_{u}\right) .
\end{aligned}
$$

Since the nonlinearities are step functions, we can simplify Eq. (6) further by constraining the form of their solutions. In particular, we look for solutions with simply connected active regions in both layers: a bump in the position layer and a stationary front in the memory layer. These assumptions lead to the following conditions: $U(x)>\theta_{u}$ for $x \in(a, b)$ and $Q(x)>\theta_{q}$ for $x \in(c, d)$. By construction, there will be two interface locations in each neural field layer $x=a, b$ in $u(x, t)$ and $x=c, d$ in $q(x, t)$. Often in studies of wave solutions to neural fields, translation invariance is used to project out one of the wave interface parameters [53]. However, in this case, the position layer $U(x)$ has translation symmetry, but the memory layer $Q(x)$ does not. Thus, to fully characterize qualitatively different solutions, we must explore all solutions $(a, b, c, d)$ to the resulting reduced equations

$$
\begin{aligned}
& U(x)=\int_{a}^{b} w_{u}(x-y) d y \\
& Q(x)=\int_{c}^{d} w_{q}(x, y) d y+\int_{a}^{b} w_{p}(x-y) d y .
\end{aligned}
$$

For specific choices of the weight kernels, the integrals in Eq. (7) can be computed explicitly, easing the identification of solutions. In particular, we use the kernels defined in Eqs. (3), (4), and (5) and evaluate integrals to find that stationary solutions take the form

$$
\begin{aligned}
& U(x)=(x-a) e^{-|x-a|}-(x-b) e^{-|x-b|}, \\
& Q(x)=\mathcal{F}(x ; c, d)+I_{0} \mathcal{P}(x ; a, b),
\end{aligned}
$$

where $\mathcal{F}$ and $\mathcal{P}$ are defined piecewise, according to the active regions $R_{q} \equiv(c, d)$ and $R_{u} \equiv(a, b)$ as

$$
\mathcal{F}(x ; c, d)=\left\{\begin{array}{lc}
\mathcal{M}_{+}(x, d)-\mathcal{M}_{+}(x, c), & x \in[d, \infty], \\
\mathcal{C}(x)-\mathcal{M}_{+}(x, c)-\mathcal{M}_{-}(x, d), & x \in[c, d], \\
\mathcal{M}_{-}(x, c)-\mathcal{M}_{-}(x, d), & x \in[-\infty, c],
\end{array}\right.
$$

where $\mathcal{C}(x)=1+\sigma \frac{\cos (n x)}{n^{2}+1}$,

$$
\mathcal{M}_{ \pm}(x, y)=\frac{e^{\mp(x-y)}}{2}\left[1+\sigma \frac{\cos (n y) \pm n \sin (n y)}{n^{2}+1}\right],
$$

and

$$
\begin{aligned}
\mathcal{P}(x ; a, b)= & \frac{1}{2}\left[\operatorname{sgn}(b-x)\left(1-e^{-\alpha|x-b|}\right)\right. \\
& \left.+\operatorname{sgn}(x-a)\left(1-e^{-\alpha|x-a|}\right)\right],
\end{aligned}
$$

where $\operatorname{sgn}(z)= \pm 1$ if $z \gtrless 0$ and $\operatorname{sgn}(0)=0$. Note that since $U(x)$ and $Q(x)$ involve integrals over the smooth weight functions $w_{u}, w_{q}$, and $w_{p}$, this mollifies the discontinuities in the Heaviside firing rates, so they will be continuously differentiable but not twice differentiable. Together with the threshold conditions $U(a)=U(b)=\theta_{u}$ and $Q(c)=Q(d)=$ $\theta_{q}$, we have implicit equations for the interface locations:

$$
\begin{aligned}
& \theta_{u}=(b-a) e^{a-b}, \\
& \theta_{q}=\mathcal{M}_{-}(c, c)-\mathcal{M}_{-}(c, d)+I_{0} \mathcal{P}(c ; a, b), \\
& \theta_{q}=\mathcal{M}_{+}(d, d)-\mathcal{M}_{+}(d, c)+I_{0} \mathcal{P}(d ; a, b) .
\end{aligned}
$$

Note that a degeneracy arises in the equation for the bump interfaces $a$ and $b$, due to the translation symmetry of the position equation, Eq. (1). Since Eq. (9) contains a mixture of transcendental functions, we do not expect to solve explicitly for vector solutions $(a, b, c, d)$. Thus, we will employ a nonlinear root finder in order to construct associated bifurcation diagrams.

We now demonstrate the mechanism by which fronts are propagated in Eq. (2), via input from the position layer, 


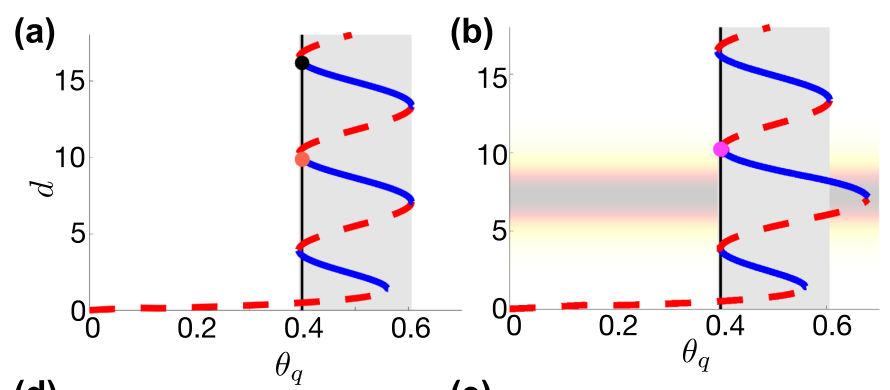

(d)

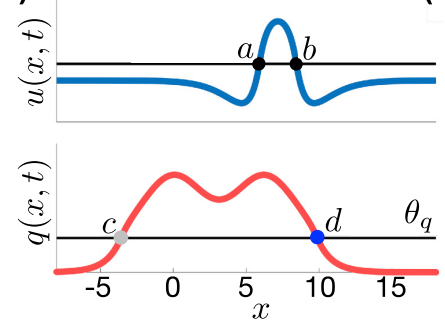

(e)

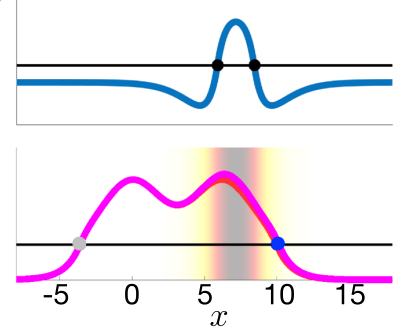

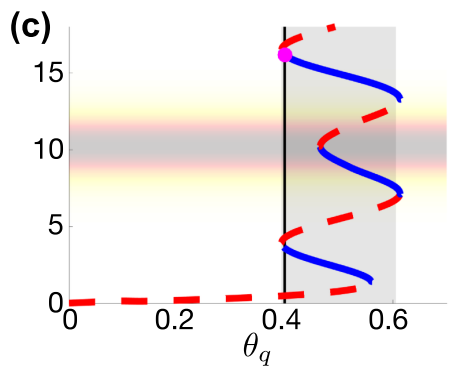

(f)

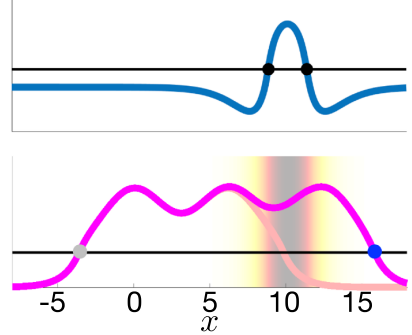

FIG. 3. Dependence of stationary solutions on the input from the position layer. (a) For no position layer input $\left(I_{0}=0\right)$, there is a range of memory layer threshold values $\theta_{q}$ (gray region), for which multiple stable (blue solid) and unstable (red dashed) standing front solutions exist. Curves represent the location of the right interface $d$ associated with the solution. Branches "snake" back and forth, turning at saddle-node (SN) bifurcations (see also Ref. [60]). The left interface $c$ is bounded to be between $-2 \pi$ and 0 (see main text), and we solve Eq. (9) to obtain the left $c$ and right interface $d$ [see panel (d)]. (b) Weak input $\left(I_{0}=0.1\right.$, background gradient) from a bump centered at $\Delta_{u}=7.184$ (near third $\mathrm{SN}$ ) shifts branches near the third SN, but weakly affects other branches. A stable solution near $d \approx 10$ for $\theta_{q}=0.4$ remains (magenta dot). (c) Weak input from bump centered at $\Delta_{u}=10.184$ (near fourth $\mathrm{SN}$ ) shifts branches near $d \approx 10$, so nearby solutions for $\theta_{q}=0.4$ vanish. [(d)-(f)] Stationary solution profiles associated with light dot in a [panel (d)]; magenta dot in b [panel (e)]; and magenta dot in c [panel (f)]. Solution from panel (d) is shown for reference in panels (e) and (f). Other parameters are $\theta_{u}=0.2, \theta_{q}=0.4, \alpha=1, n=1, \sigma=0.3$.

Eq. (1). This analysis uses bifurcation diagrams associated with stationary solutions, but in Sec. IV we approximate the dynamics of the bump and front interfaces to obtain a low-dimensional system for the motion of the patterns in each layer. Bifurcation curves and stationary solutions of the model Eq. (1), (2) are shown in Fig. 3. Nonlinear root finding applied to Eq. (9) is used to compute the bifurcation curves, but the stability will be determined by a linear analysis below. To clearly display solution curves, we have bounded the left interface $c$ of the front solution between $[-2 \pi, 0]$. Note that similar bifurcation curves would be obtained by bounding $c \in[-2(m+1) \pi,-2 m \pi]$ for any positive integer $m$. The location of the left interface $c$ only marginally affects the right interface, since interactions between the interfaces are described by the function $e^{c-d}$ in Eq. (9), which will typically be small. In Ref. [60], this was addressed by plotting bifurcation diagrams showing the dependence of the width $L_{f}=d-c$ of the front, rather than the right interface $d$. For our purposes, it is more instructive to track how $d$ changes with the location of the bump in the position layer.

The stationary solutions' dependence on the memory layer threshold $\theta_{q}$ and input from the position layer is shown in Figs. 3(a)-3(c). The case of no input $\left[I_{0} \equiv 0\right.$ in Eq. (9) $]$ is shown in Fig. 3(a). Note the metastability of solution in the gray shaded region. Advancing the front interface to subsequent stable branches is the main mechanism by which previously visited locations are stored by the network. When input from the position layer is applied to the memory layer [Figs. 3(b) and 3(c)], it warps the solution curves in the vicinity of the excitation. This can result in the annihilation of stable solutions at lower values of $\theta_{q}$ [Fig. 3(c)]. We plot profiles in Figs. 3(d)-3(f), demonstrating how solutions are identified with their threshold intersection points. Note input from the position layer is not sufficient to destabilize the input-free solution in Fig. 3(e), but is in Fig. 3(f) since the input is slightly ahead of the front interface. This is the mechanism by which the memory layer's front is propagated, once moving bumps in the position layer are considered: The bump must be ahead of the front interface to propagate it forward.

Next, we take a closer look at the bifurcation that occurs by increasing the strength $I_{0}$ of the input from the position layer to the memory layer. In particular, we consider a one-sided front, as this a fairly accurate approximation of the case $d-c \gg 1$, and we will utilize this observation in our low-dimensional system we derive in Sec. IV.

\section{B. One-sided front}

Terms involving $e^{c-d}$ will tend to be quite small, even for a modest difference between the two front interfaces (e.g., $\left.e^{-10} \approx 4.54 \times 10^{-5}\right)$. Thus, we consider the case where $c-d$ is sufficiently large as to ignore the exponentially small term $e^{c-d}$, and focus specifically on using Eq. (9) to solve for the right interface $d$. In this case, we can write

$$
\begin{aligned}
& \theta_{u}=(b-a) e^{a-b}, \\
& \theta_{q}=\frac{1}{2}+\frac{\sigma \cos (n d)+n \sigma \sin (n d)}{2\left(n^{2}+1\right)}+I_{0} \mathcal{P}(d ; a, b),
\end{aligned}
$$

so that Eqs. (10a) and (10b) can be solved in sequence to obtain bifurcation curves for $d$, similar to Fig. 3, except for small differences arising for low values of $d$. As we also 

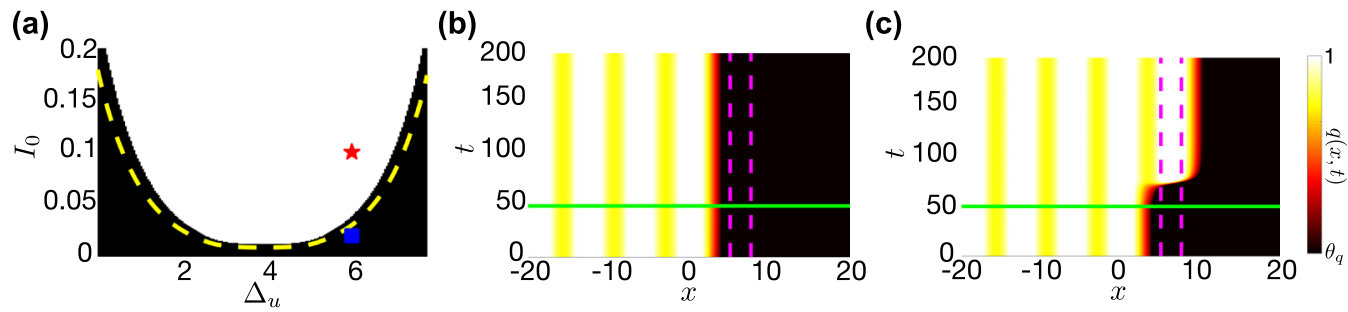

FIG. 4. Phase diagram showing the impact of the bump location $\Delta_{u}$ and input amplitude $I_{0}$ on the movement of a nearby front interface. (a) Partition of $\left(\Delta_{u}, I_{0}\right)$ parameter space into regions where front propagates forward (white) and where it does not (black). Line shows analytical approximation derived from Eq. (10) (see Appendix A). Mismatch arises due to dynamics' strong sensitivity to discretization errors in the numerical scheme for solving Eqs. (1) and (2). [(b), (c)] Numerical simulations of the full network, Eqs. (1) and (2), with a stationary bump centered at $\Delta_{u}=6$ (interfaces given by dashed lines). The position input amplitude is switched from $I_{0}=0$ to $I_{0}=0.02$ in panel (b) [blue square in panel (a)] and $I_{0}=0.1$ in panel (c) [red star in panel (a)] at $t=50$ (light line). The front propagates for large enough $I_{0}$ [panel (c)]. Color gradient denotes the values of $q(x, t)$ in space $x$ and time $t$. Other parameters are $\theta_{u}=0.2, \theta_{q}=0.4, \sigma=0.3, n=1$, and $\alpha=1$.

demonstrated in Fig. 3(c), increasing the strength of the input $I_{0}$ from the position layer to the memory layer can lead to the annihilation of the pair of stable-unstable solutions via a saddle-node $(\mathrm{SN})$ bifurcation. We derive this curve of $\mathrm{SN}$ bifurcations in Appendix A, identifying the point at which the bend of the bifurcation curves in Fig. 3 cross through a threshold value $\theta_{q}$ due to an increase of the input $I_{0}$.

We compare our analytical results to numerical simulations in Fig. 4. In particular, we study the input strength $I_{0}$ necessary to advance the location of the front in the memory layer to the next stable branch of solutions [see Figs. 3(b) and 3(c) for further illustration]. As the location $\Delta_{u}$ of the bump in the position layer is varied, the critical input strength $I_{0}^{c}$ at which the front in the memory layer advances varies nonmonotonically [Fig. 4(a)]. At an intermediate value of the bump location, $I_{0}^{c}$ obtains a minimum. Thus, activity in the memory layer propagates best when the bump is slightly advanced as compared to the front location. Our results agree well with numerical simulations. We demonstrate examples of the front dynamics in the full systems, Eqs. (1), (2), in Figs. 4(b) and 4(c). When $I_{0}<I_{0}^{c}$, the front does not advance to the next stable branch, but the front does advance if $I_{0}>I_{0}^{c}$.

Next, we examine the linear stability of the solutions computed above. In particular, we expect the eigenvalues of front solutions $Q(x)$ to equal zero at the $\mathrm{SN}$ bifurcation points. Such solutions indicate boundaries at which $I_{0}$ can be changed to propagate the front from one stable branch to the next.

\section{Linear stability}

The stability of stationary solutions, defined by Eq. (9), can be determined by examining the evolution of small smooth perturbations. This allows us to derive a set of linearized equations, which can be analyzed to yield eigensolutions that indicate the stability of the stationary bump and fronts derived above.

We solve for the eigenvalues associated with linear stability in Appendix B and plot the result in Fig. 5(a). The eigenvalue $\lambda_{d}$ corresponds to the response of fronts to translating perturbations applied to the right front interface, close to where the input from the bump layer is received. As expected, the eigenvalue becomes zero at the endpoints of the stable branch, annihilating in a SN bifurcation. Eigenfunctions are determined directly from our linearized equations, after solving for the eigenvalues (see Appendix B). The eigenfunction associated with $\lambda_{d}$ is plotted in Fig. 5(b), showing perturbations shift the location of the right front interface to the right.

With knowledge of the mechanism by which the position layer $u(x, t)$ moves the memory layer front $q(x, t)$, we now derive low-dimensional approximations of the bump and front motion. Our interface calculations track the location of the bump $\Delta_{u}(t)$ as well as the left and right locations of the memory front $\Delta_{+}(t)$ and $\Delta_{-}(t)$ using a system of three nonlinear differential equations.

\section{INTERFACE EQUATIONS}

We now derive interface equations for the position layer $u(x, t)$ and the front layer $q(x, t)$, starting with mild assumptions on the parameters and dynamics of the activity in each layer. Strong assumptions of weak heterogeneity and inputs will be used to simplify the form of our derived interface equations. Interface equations reduce the dimensionality of
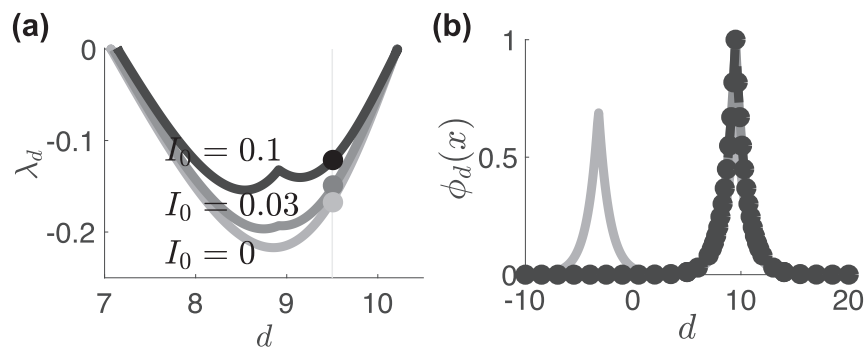

FIG. 5. Eigensolution for perturbations near the right interface of the front at $x=d$. (a) Eigenvalue varies nonmonotonically for different values of $\theta_{q}$ (correspondingly plotted vs $d$ ) along the stable branch corresponding to those $d$ values. As input amplitude $I_{0}$ is increased, the range of $d$ values decreases and $\lambda_{d}$ moves closer to zero (see Appendix B). Cusp arises at boundary of input (bump in position layer is centered at $\Delta_{u}=10.184$ ). (b) Eigenfunction $\phi_{d}(x)$ associated with perturbations of the right interface, $x=d$ (see Appendix B). Peak near $x=d$ increases as the input amplitude is increased $I_{0}$. Shades correspond to input strength $I_{0}$ as in panel (a) Other parameters are $\sigma=0.3, \alpha=1, n=1, c \in[-2 \pi, 0]$, and $\theta_{u}=0.2$. 
our system due to the Heaviside form of the nonlinearities in Eqs. (1) and (2), so that the threshold crossing points $u(x, t)=\theta_{u}$ and $q(x, t)=\theta_{q}$ largely determine the dynamics of the full system. Several authors have extended the seminal work of Amari [48], who developed interface methods for analyzing bump stability in neural fields, to handle more complicated dynamics like fronts in heterogeneous networks [59] and planar domains [61]. The details of the derivation of the interface equations are given in Appendix C.

We can reduce the dynamics of Eqs. (1) and (2) to an integral for the location of the bump, $\Delta_{u}(t)=\int_{0}^{t} v(s) \mathrm{d} s+\Delta_{u}(0)$, and a pair of nonlinear differential equations for the right $\left[\Delta_{+}(t)\right]$ and left $\left[\Delta_{-}(t)\right]$ front interfaces:

$$
\begin{aligned}
\frac{d \Delta_{+}}{d t}= & \frac{1}{\left|Q^{\prime}(d)\right|}\left[\sigma \frac{\cos \left[n \Delta_{+}(t)\right]+n \sin \left[n \Delta_{+}(t)\right]}{2\left(n^{2}+1\right)}\right. \\
& \left.+I_{0} G\left(\Delta_{+}(t)-\Delta_{u}(t)\right)+\frac{1}{2}-\theta_{q}\right], \\
\frac{d \Delta_{-}}{d t}= & -\frac{1}{\left|Q^{\prime}(d)\right|}\left[\sigma \frac{\cos \left[n \Delta_{-}(t)\right]-n \sin \left[n \Delta_{-}(t)\right]}{2\left(n^{2}+1\right)}\right. \\
& \left.+I_{0} G\left(\Delta_{-}(t)-\Delta_{u}(t)\right)+\frac{1}{2}-\theta_{q}\right],
\end{aligned}
$$

where $G(\Delta)=\mathcal{S}(h-\Delta)+\mathcal{S}(\Delta+h)$ and $\mathcal{S}(x)=\operatorname{sgn}(x)$ $\left(1-\mathrm{e}^{-\alpha|x|}\right)$, and

$$
\left|Q^{\prime}(d)\right|=\frac{1}{2}\left[1+\sigma \frac{\cos (n d)+n \sin (n d)}{n^{2}+1}\right] .
$$

We note a number of features of the full system Eqs. (1) and (2) captured by the interface Eq. (11). First, in the absence of any heterogeneity $(\sigma=0)$ or positional input $\left(I_{0}=0\right)$, the front interfaces propagate at a speed approximated by $\gamma_{d}\left(1 / 2-\theta_{q}\right)$ on the right $\left[\Delta_{+}(t)\right]$ and $\gamma_{c}\left(1 / 2-\theta_{q}\right)$ on the left $\left[\Delta_{-}(t)\right]$. Sufficiently strong heterogeneity $\left(\sigma=\sigma^{c}>0\right)$ will pin the front. Without any positional input $\left(I_{0}=0\right)$, the critical value $\sigma^{c}$ that pins fronts is given by the $\sigma$ such that the maximum of $\sigma\left[\cos \left(n \Delta_{+}\right)+n \sin \left(n \Delta_{+}\right)\right]$equals $n^{2}+1-2 \theta_{q}$. This occurs when $\sigma^{c}=\left[n^{2}+1-2 \theta_{q}\right] /[\cos \mathcal{T}(n)-n \sin \mathcal{T}(n)]$ for $\mathcal{T}(n)=2 \tan ^{-1}\left[\left(1-\sqrt{n^{2}+1}\right) / n\right]$, corresponding to the critical heterogeneity for wave propagation failure discussed in Refs. [58,59]. Thus, we require $\sigma>\sigma^{c}$ for the system to retain memory of visited locations, which prevents front propagation to the rest of the domain.

Our interface equations are compared with simulations of the full model Eqs. (1) and (2) in Fig. 6. The evolution of the bump interfaces in the positional layer $u(x, t)\left[u\left(x_{ \pm}(t), t\right)=\theta_{u}\right]$ are captured well by $x_{ \pm}(t)=\Delta_{u}(t) \pm h$ [Figs. 6(a) and 6(c)]. We expect the mismatch arises as the result of our static gradient approximation $u_{x}\left(x_{ \pm}(t), t\right) \approx \pm U^{\prime}( \pm h)$. The front tracks previously visited locations of the bump, corresponding to the active regions in the domain at time $t$ [Figs. 6(b) and $6(\mathrm{~d})$ ]. More regions are activated when the searcher position enters an unvisited part of the domain. Otherwise, the front solution remains stationary. Thus far, we have utilized an open-loop velocity protocol, so that the velocity input to the position layer does not receive feedback from the memory layer.

Our low-dimensional approximation, Eq. (11), agrees with numerical simulations. Thus, we have established a (a)

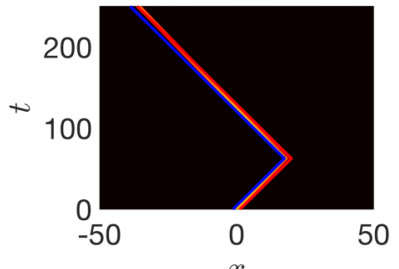

(b)

$x$

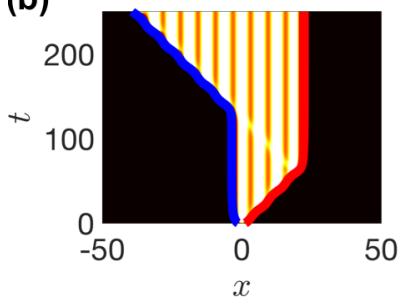

(c)

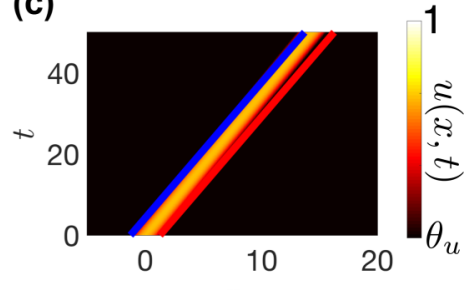

(d)

$x$

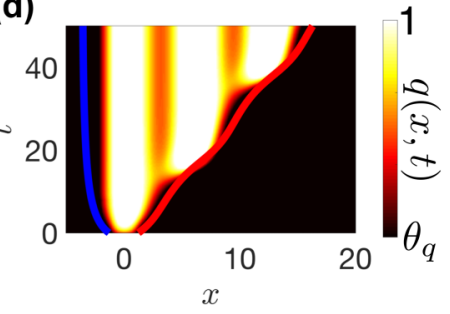

FIG. 6. Interface equations approximate the dynamics of the full neural field model, Eqs. (1) and (2). (a) Bump propagates across the domain of the position layer, $u(x, t)$, in response to a velocity input defined $v(t)=0.3$ on $t \in[0,62.5)$ and $v(t)=-0.3$ on $t \in$ [62.5,250]. Interfaces approximation $\Delta_{u}(t) \pm h$ (see Appendix C) agrees well with the threshold crossing locations $u\left(x_{ \pm}(t), t\right)=\theta_{u}$ of the full simulation, Eqs. (1) and (2). (b) Memory layer, $q(x, t)$, supports a front solution that propagates in response to the motion of the bump in the position layer. Our interface approximation, $\Delta_{ \pm}(t)$, given by Eq. (11) correspondingly tracks the left and right boundaries of the visited regions of the searching agent. [(c), (d)] Enlarged versions of the simulations in panels (a) and (b), showing slight mismatches in the approximation that occur due to our truncations. Color gradient denotes the values of $u(x, t)$ and $q(x, t)$ in space $x$ and time $t$. Parameters are $\theta_{u}=0.2, \theta_{q}=0.4, n=1, \sigma=0.3, I_{0}=0.2$, and $\alpha=1$.

mechanism by which a ballistic searcher may store a memory of previously visited locations. In the next section, we analyze our reduced model and study search strategies whereby an agent's behavior depends on which regions it has searched.

\section{MEMORY-GUIDED SEARCH}

Now, we study the impact of memory-guided control on the efficiency of a searching agent with memory of previously visited locations. We measure efficiency by calculating the mean first passage time associated with absorption into the target, which is commonly used to quantify search efficiency, presuming the agent uses more resources with time [13,15]. More complicated measures of search efficiency are possible, such as penalizing high search speeds, but we focus on the simple passage time measure in this initial study. First, we consider search along a single segment, and control speeds up search when the agent arrives at a previously visited location. Assuming stochastic discovery of targets, this reduces time spent in previously visited locations. Interestingly, this does not appear to reduce search time in single segments, and the optimal search speed is the same whether in novel or previous searched territory. Second, we consider a radial arm maze, changing the geometry from a single segment to multiple segments connected at one end. An IOR strategy is 

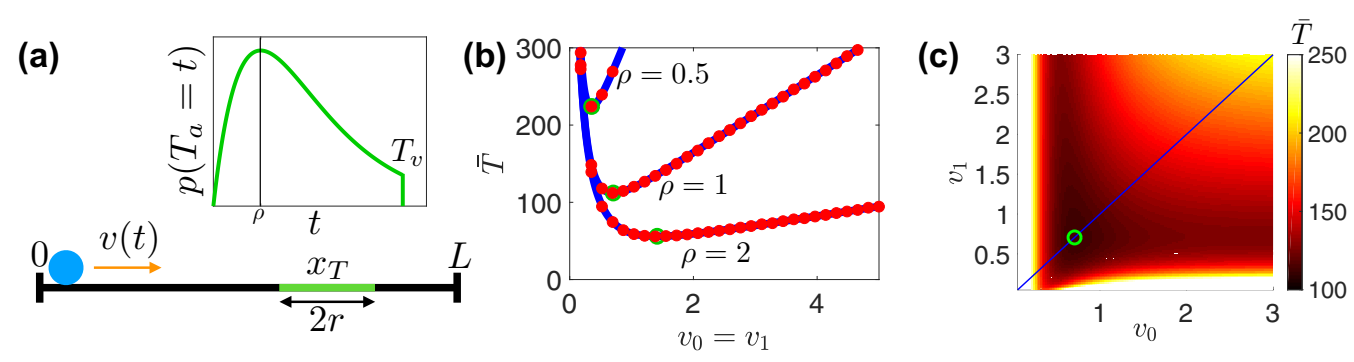

FIG. 7. Ballistically moving agent searches for a hidden target. (a) Searcher (dot) begins at the left edge $(x=0)$ of the domain $(x \in[0, L])$, initially moving with speed $v_{0}$ and then moving with speed $v_{1}$ on all subsequent trips across. The target spanning $x \in\left[x_{T}-r, x_{T}+r\right]$ is stochastically discoverable according to the waiting time density $p(t)=\rho^{2} t \mathrm{e}^{-\rho t}$ (plot above), so if the waiting time exceeds $T_{v}$, the searcher will not find the target on the current trip. (b) Plots of $\bar{T}$ vs $v_{0}=v_{1}$ (line) using Eq. (15) are nonmonotonic, revealing an interior optimum that minimizes the average search time (circles). As the rate of target discovery $\rho$ decreases, $\bar{T}$ increases and the optimal $v_{0}$ decreases. Theory matches well with averages from $10^{6}$ Monte Carlo simulations (dots). (c) Mean search time $\bar{T}$ as a function of both $v_{0}$ and $v_{1}$, showing the optimal choice $\left(v_{0}, v_{1}\right)$ occurs when $v_{0}=v_{1} \approx 0.706$ (circle) when $\rho=1$. Other parameters are $L=100$ and $r=1$.

advantageous in this case, since it prevents the agent from searching previously searched arms, in the initial exploratory phase of the search. Our theory is compared to Monte Carlo simulations of ballistic searcher model, which we describe below.

\section{A. Single segment}

We begin by exploring memory-guided search in a single segment. Avoiding previously visited locations will not work in this case, since the searcher would be trapped at the end of the segment, so we invoke a strategy whereby the searcher changes speed when exploring a previously visited portion of the segment. In Appendix D, we show that the effects of the velocity feedback in the control term $v(t)=\chi(u, q) \bar{v}(t)$ can be mapped to a ballistically moving particle model of a searcher. Effectively, we have reduced the neural field model, Eqs. (1) and (2), to the interface Eqs. (11), which can further be approximated by a particle model with switching speeds. With this in mind, we can map the dynamics of memory-guided search to a much simpler model we describe below.

We model the agent's search behavior as follows. The agent enters a bounded interval $x \in[0, L]$ from the left side $(x=0)$ and searches ballistically at a constant speed, determined by whether it is in the unsearched $\left(v_{0}\right)$ or searched domain $\left(v_{1}\right)$. The finite-sized target has radius $r$ and is centered at $x_{T}$, so it spans $x \in\left[x_{T}-r, x_{T}+r\right]$ and $r \leqslant x_{T} \leqslant L-r$ [Fig. 7(a)]. The agent discovers the target stochastically, according to a $\Gamma$ distributed waiting time, $\Gamma(t, \rho)=\rho^{2} \mathrm{e}^{-\rho t}$. This is motivated by the experimental observation that reaction times for visual search over a small number of items are $\Gamma$ distributed [62]. Thus, we think of this as a local approximation of the item discovery dynamics. Such dynamics could arise for a two-stage process whereby the searcher first realizes an object of interest is nearby and then compares it with the target object from memory. For velocity $v$, the agent is over the target for a time $T_{v}=2 r / v$, so the probability of discovering the target on a single trip is

$$
P_{v}=\rho^{2} \int_{0}^{T_{v}} t e^{-\rho t} d t=1-\left(1+\rho T_{v}\right) e^{-\rho T_{v}},
$$

and the associated conditional mean time to find the target while over it is

$$
\begin{aligned}
T_{a}(v) & =\frac{\rho^{2}}{P_{v}} \int_{0}^{T_{v}} t^{2} e^{-\rho t} d t \\
& =\frac{1}{\rho P_{v}}\left[2-\left(2+2 \rho T_{v}+\rho^{2} T_{v}^{2}\right) e^{-\rho T_{v}}\right] .
\end{aligned}
$$

We now address the problem of finding the velocities $\left(v_{0}, v_{1}\right)$, corresponding to the novel and searched territory, that minimize the time to find the target. The mean first passage time can be derived analytically by tracking the probability of absorption and accumulated search time at each target encounter. The calculation is presented in detail in Appendix D. Ultimately, we find an expression for the generalized mean first passage time in terms of the search velocities $\left(v_{0}, v_{1}\right)$ and domain length $L$, renormalized by the target radius

$$
\begin{aligned}
\bar{T}= & \frac{L-2}{2 v_{0}}+P_{v_{0}} T_{a}\left(v_{0}\right)+\left(1-P_{v_{0}}\right)\left[\frac{L}{v_{1} P_{v_{1}}}\right. \\
& \left.+\left(1+\frac{L}{2}\right)\left(\frac{1}{v_{0}}-\frac{1}{v_{1}}\right)+T_{a}\left(v_{1}\right)\right] .
\end{aligned}
$$

Note, for constant speeds $v_{1}=v_{0}$, Eq. (14) simplifies considerably to

$$
\bar{T}\left(v_{1} \equiv v_{0}\right)=\frac{L}{2 P_{v_{0}} v_{0}}\left(2-P_{v_{0}}\right)+T_{a}\left(v_{0}\right)-\frac{1}{v_{0}} .
$$

As shown in Fig. 7(b), $\bar{T}\left(v_{1}=v_{0}\right)$ has an internal minimum, which leads to the most rapid finding of the target. Notably, in Fig. 7(c), we find there is no advantage in searching more quickly (or slowly), once the domain has already been searched: The search time is minimized when $v_{1}=v_{0}$.

Thus, for single segments, memory guidance does not speed up search in this particular paradigm. The optimal strategy for minimizing the time to find the target is for the searcher to maintain the same search speed throughout the exploration process. We now demonstrate an alternative paradigm in which memory-guided search does reduce the time to find the target.

\section{B. Radial arm maze}

Since search on a single segment is not aided by memory guidance, we examine the case in which the agent must search over a space with more complex topology. In particular, we 
(a)

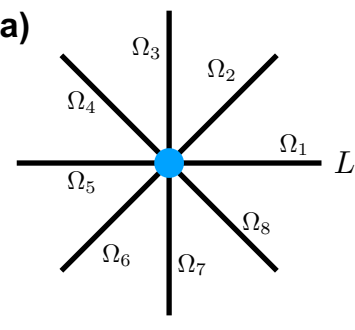

(b)

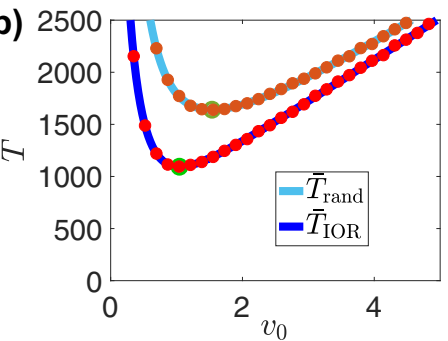

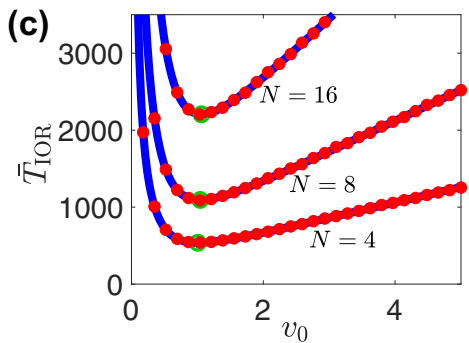

FIG. 8. Ballistically moving agent searches a radial arm maze with a single target in a single arm. (a) Searcher (dot) begins at the center of the maze and chooses a random arm $\Omega_{k}, k \in\{1, \ldots, N\}$ to search. Purely random search (rand) proceeds with the searcher always choosing 1 out of $N$ total arm. Inhibition of return (IOR) guides searcher away from previously searched arms, so the first $N$ arms chosen are the arms $k=1, \ldots, N$. (b) Inhibition of return leads to more rapid location of the target than purely random search $\left[\bar{T}_{\text {rand }}>\bar{T}_{\mathrm{IOR}}\right.$ as in Eq. (19)]. Theory (solid lines) matches $10^{6}$ Monte Carlo simulations (dots) very well. Here $N=8$. (c) Average time to find the target $\bar{T}_{\mathrm{IOR}}$ using IOR increases with the number of arms, but note the optimal search speed $v_{0}^{\min }$ (circles) remains relatively unchanged. Other parameters are $L=100, r=1$, and $\rho=1$.

study the problem of the searcher finding a hidden target in a radial arm maze [Fig. 8(a). This paradigm has been used to test mammalian memory, requiring a combination of spatial navigation, decision making, and working memory $[63,64]$. Rather than deriving a new neural field model and associated interface equations on this more complex domain, we develop a simpler model for memory of previously visited locations using a metastable neuronal network with distinct populations encoding each arm. The searcher begins at the center of the maze with $N$ arms that radiate outward, so locations lie on the union of bounded intervals $\Omega_{1} \cup \Omega_{2} \cup \cdots \cup \Omega_{N}$ with $\Omega_{j}=[0, L]$ for all $j$. The target lies within one of the arms $k \in$ $\{1, \ldots, N\}$ at a location $x_{T} \in[r, L-r]$ as before. Since our previous analysis did not reveal an advantage to storing the spatial structure of locations visited within a segment, we model memory of visited arms by distinct bistable neural populations:

$$
\dot{q}_{j}(t)=-q_{j}(t)+H\left(q_{j}-\theta_{q}\right)+I_{j}(t),
$$

where $I_{j}(t)=I_{0}>\theta_{q}$ when the agent visits arm $j$ and $I_{j}(t)=0$ otherwise. The variables $q_{j}(t) \rightarrow 1$ once $\operatorname{arm} j$ is visited, and initially $q_{j}(0)=0$ for all $j$. If the searcher avoids arms such that $q_{j}(t)>\theta_{q}$, they will only visit novel arms until $q_{j}(t) \rightarrow 1$ for all $j$. Thus, Eq. (16) constitutes a discretized version of Eq. (2). When the searcher is over the target, it discovers it according to a $\Gamma$ distributed waiting time. The probability of discovering the target at each encounter is $P_{v}$, Eq. (12), and the conditional mean first passage time within the target is $T_{a}(v)$, Eq. (13).

We now derive the mean time to find the target, as in the case of a single armed domain. In particular, we compare the effects of IOR, where the searcher avoids previously explored arms initially, as opposed to a memoryless selection of the next arm to be searched. As mentioned, we assume the speed of search is constant throughout the process $|v(t)| \equiv v_{0}$ for all $t$. Following the steps of our previous calculation (and see also Ref. [65]), we find that (for $r=1$ ) the average time for a memoryless searcher to find a target placed uniformly on $x_{T} \in[1, L-1]$ on one of $N$ radial arms is

$$
\begin{aligned}
\bar{T}_{\text {rand }}= & \frac{2 L(N-1)}{v_{0}}+\frac{2 N L\left(1-P_{v_{0}}\right)^{2}}{P_{v_{0}}\left(2-P_{v_{0}}\right) v_{0}} \\
& +\frac{L\left(1-P_{v_{0}}\right)}{\left(2-P_{v_{0}}\right) v_{0}}+\frac{L-2}{2 v_{0}}+T_{a}\left(v_{0}\right) .
\end{aligned}
$$

On the other hand, a searcher that uses IOR to avoid previously explored arms prior to all arms being searched finds the target after an average time

$$
\begin{aligned}
\bar{T}_{\mathrm{IOR}}= & \frac{L(N-1)}{v_{0}}+\frac{2 N L\left(1-P_{v_{0}}\right)^{2}}{P_{v_{0}}\left(2-P_{v_{0}}\right) v_{0}} \\
& +\frac{L\left(1-P_{v_{0}}\right)}{\left(2-P_{v_{0}}\right) v_{0}}+\frac{L-2}{2 v_{0}}+T_{a}\left(v_{0}\right),
\end{aligned}
$$

which appears nearly the same as the random search time $\bar{T}_{\text {rand }}$, except that the leading factor is roughly half for $\bar{T}_{\mathrm{IOR}}$. In fact, $\bar{T}_{\text {rand }} \geqslant \bar{T}_{\text {IOR }}$ for any $N \geqslant 2$, since

$$
\bar{T}_{\text {rand }}-\bar{T}_{\mathrm{IOR}}=\frac{L(N-1)}{v_{0}}>0,
$$

for $N \geqslant 2$. This theory agrees with Monte Carlo simulations of the ballistic searcher [Fig. 8(b)], demonstrating the efficacy of IOR in reducing the time to find the target. This effect is stronger for mazes with more arms (higher $N$ ) as the total time to find the target [Fig. 8(d)] and the discrepancy between IOR and random search increases with $N$. Note these results do not depend strongly on the particular form of the distribution of waiting times, as long as it is a unimodal peaked distribution. However, for purely decreasing distributions like an exponential, the optimal search strategy is to take the search velocity $v_{0} \rightarrow \infty$ which is unrealistic.

Our analysis of the neural field model has demonstrated a plausible neural mechanism for memory-guided search, persistent activity encoding previously searched regions. The theory and simulations we have performed here for ballistic searcher models has demonstrated that memory-guided search does not appear to be advantageous in one-dimensional domains composed of a single segment. However, multiple segments adjoined at there ends can comprise more complex domains like the radial arm maze, which do benefit from inhibition of return (IOR). A searcher that avoids previously searched segments will tend to find a randomly placed target more quickly than a searcher that chooses subsequent arms in a memoryless way. Since our low-dimensional theory was derived from the full neural field equations, we expect that stochastic simulations of the full neural field model would yield qualitatively similar results. We discuss briefly how our theory might be extended to planar domains. 

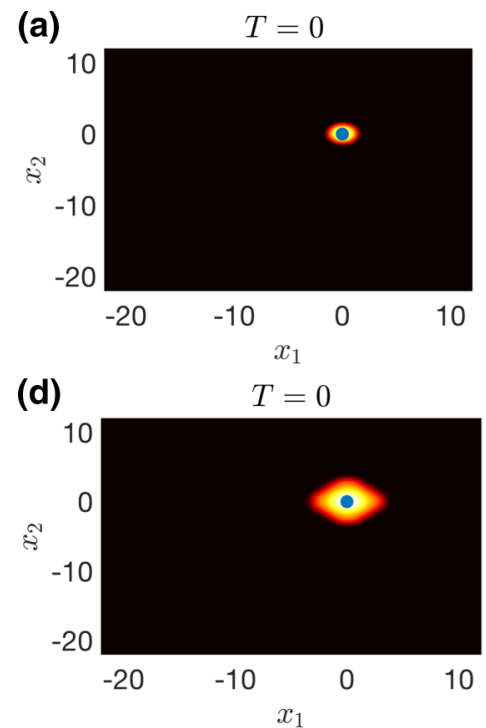

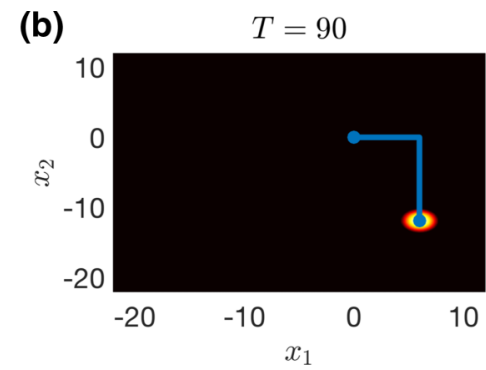

(e)

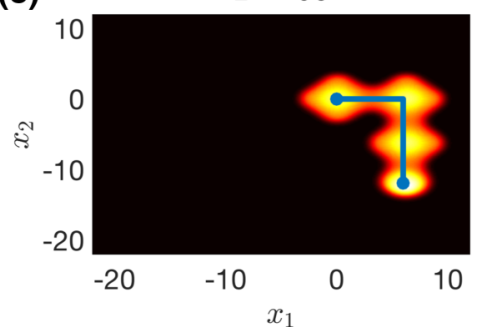

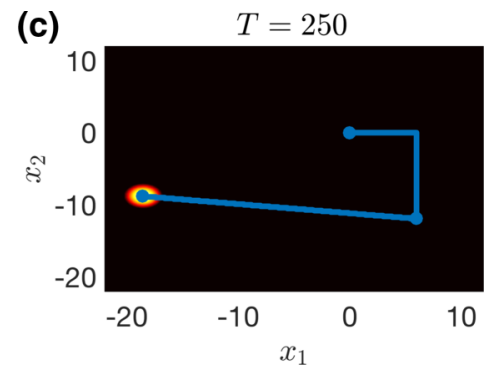

(f)

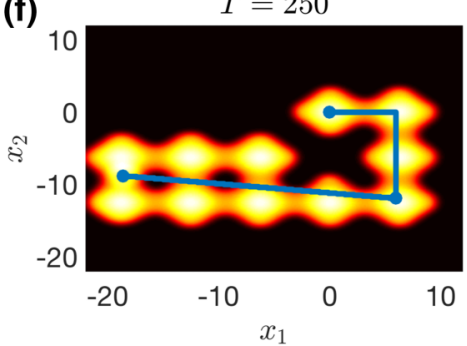

FIG. 9. Two-dimensional simulation of the neural field model, Eq. (20), describing the propagation of a coupled bump and front in a planar domain. Evolution of the bump in the position layer $u(\mathbf{x}, t)$ is tracked by showing snapshots at (a) $T=0$; (b) $T=90$; and (c) $T=250$. The path of the agent is shown by the solid blue line. Motion of the bump layer is stored by the front layer $q(\mathbf{x}, t)$, which tracks the previously visited locations of the bump at the same snapshots in time: (d) $T=0$; (e) $T=90$; and (f) $T=250$. Parameters are $\theta_{u}=0.2, \theta_{q}=0.45$, $n=1, \sigma=0.3, v=0.3$, and $I_{0}=0.3$.

\section{EXTENSIONS TO TWO DIMENSIONS}

Most visual and navigational search tasks tend to be in spaces of two or more dimensions (see Figs. 1(d) and 1(e) and Refs. [1-3]). In future work, we will extend our analysis of our one-dimensional model, Eqs. (1) and (2), to an analogous two-dimensional model. We expect there to be a wider variety of control mechanisms that lead to an efficient use of memory in guiding future search locations. Analysis of stationary solutions in planar neural field models has been successful in a number of cases [66-69], and there is a clear path to extending interface methods to describe contour boundaries that arise for solutions in planar systems [61].

Here we discuss a candidate model for memory-guided search in two dimensions. In particular, we will demonstrate in numerical simulations that such a model does result in a model that can store previously visited locations in the plane. Memory of a searching agent's position and memory for previously searched locations are captured by the following pair of neural field equations on a planar domain:

$$
\begin{aligned}
& u_{t}=-u+w_{u} * H\left(u-\theta_{u}\right)-\mathbf{v}(t)\left(\nabla w_{u}\right) * H\left(u-\theta_{u}\right), \\
& q_{t}=-q+w_{q} * H\left(q-\theta_{q}\right)+w_{p} * H\left(u-\theta_{u}\right)
\end{aligned}
$$

defined on $\mathbf{x}=\left(x_{1}, x_{2}\right)^{T} \in \mathbb{R}^{2}$. Recurrent coupling in the position layer is described by the integral $w_{u} * H\left(u-\theta_{u}\right)=$ $\int_{\mathbb{R}^{2}} w_{u}(\mathbf{x}-\mathbf{y}) H\left(u(\mathbf{y}, t)-\theta_{u}\right) d \mathbf{y}$, and the synaptic kernel is lateral inhibitory and rotationally symmetric $\left(w_{u}(\mathbf{x}, \mathbf{y})=w_{u}(z)\right.$, $z=\sqrt{\left.\left(x_{1}-y_{1}\right)^{2}+\left(x_{2}-y_{2}\right)^{2}\right)}$ comprised of a difference of Bessel functions of the second kind [68]:

$$
w_{u}(z)=\sum_{k=1}^{4} c_{k} K_{0}\left(\alpha_{k} z\right)
$$

with $\left[c_{1}, c_{2}, c_{3}, c_{4}\right]=[5 / 3,-5 / 3,-1 / 2,1 / 2]$ and $\left[\alpha_{1}, \alpha_{2}, \alpha_{3}, \alpha_{4}\right]$ $=[1,2,1 / 4,1 / 2]$. Velocity input is given by a two-dimensional vector $\mathbf{v}(t)=\left[v_{1}(t), v_{2}(t)\right]^{T}$, which translate bumps when taking its dot product with the gradient of the weight function $\nabla w_{u}(r)=\left(\partial_{x_{1}}, \partial_{x_{2}}\right)^{T} w_{u}(r), r=\sqrt{x_{1}^{2}+x_{2}^{2}}$. The heterogeneous connectivity function that pins the activity in the memory layer is defined using the product of cosines and an exponential:

$$
w_{q}(\mathbf{x}, \mathbf{y})=\left[1+\sigma \cos \left(n y_{1}\right)+\sigma_{2} \cos \left(n y_{2}\right)\right] \frac{e^{-v d^{2}}}{2 \pi} .
$$

As in the one-dimensional case, the weight function is a homogeneous kernel modulated by periodic heterogeneities. We will demonstrate in numerical simulations that these heterogeneities can pin the expansion of wave fronts, analogous to the stabilizing effects they have on stationary bumps in planar neural fields [70]. Lastly, we consider an input term from the position layer, applying feedforward input centered at the location of the bump:

$$
w_{p}(d)=I_{0} e^{-v z^{2}} .
$$

We now demonstrate that this model is capable of generating a memory trace for previously visited regions of a searcher exploring two-dimensional space. In Fig. 9, we demonstrate the results from a numerical simulation of the neural field, Eq. (20). A bump is instantiated in the position layer $u(\mathbf{x}, t)$, and tracks the locations visited by an agent moving about the domain [Figs. 9(a)-9(c)], evolving in response to velocity inputs. The motion of the bump is reflected by the memory of previously visited locations tracked by the front layer $q(\mathbf{x}, t)$ [Figs. 9(d)-9(f)]. The activity in the front layer is stabilized by the heterogeneity in the weight kernel, Eq. (21), as it was in the one-dimensional case.

We now discuss a control mechanism that we conjecture could lead to successful inhibition of return of a searcher with position and memory layer activity described by Eq. (20). In 
(a)

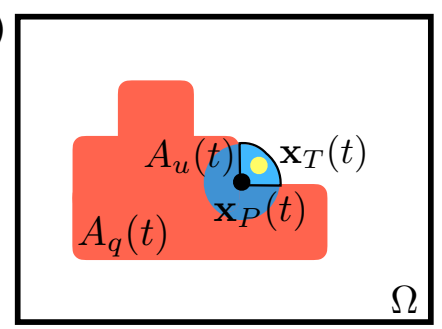

(b)

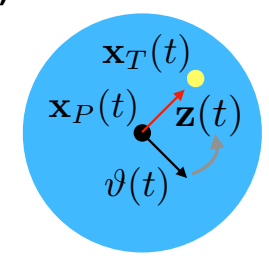

FIG. 10. Inhibition of return is implemented when an agent moves in the direction of the unexplored regions of the domain $\Omega$. (a) The intersection of the bump layer's active region $A_{u}(t)$ and the complement of the active region $A_{q}(t)$ is the region yet to be searched (quarter section of circle) with center-of-mass $x_{T}(t)$ close to the agent's current location $\mathbf{x}_{P}(t)$ (black dot). (b) The agent reorients its velocity angle $\vartheta(t)$ in the direction of the unexplored region $\left[\mathbf{x}_{T}(t)\right]$.

particular, the position and memory layers will have active regions $A_{u}(t)$ and $A_{q}(t)$ describing the area of superthreshold within each [Fig. 10(a) $\}$. From the active region $A_{u}(t)$, the position center-of-mass $\mathbf{x}_{P}(t)$ can be computed as the first moment. Second, the region $A_{u}(t) \cap\left[\Omega \backslash A_{q}(t)\right]$ describes where the position layer's activity intersects with the complement of the memory layer's activity, corresponding to unexplored space. If we call the center of mass of this region $\mathbf{x}_{T}(t)$, then an IOR mechanism might work by having the searcher move toward $\mathbf{x}_{T}(t)$. Thus, the angle of the searcher's velocity $\vartheta(t)$ should constantly orient in the direction of $\mathbf{x}_{T}(t)$ [Fig. 10(b)].

Note that the computations involved in determining a gradient-descent type orientation of the searcher require some linear readouts [71], divisive normalization [72,73], and nonlinearities. Motor control circuits are capable of producing outputs that correspond to a wide range of nonlinearities, for example, built on summations of various nonlinear basis functions [74]. Thus, we expect the computation we have outlined above could be implemented as a closed-loop feedback from the memory system onto a corresponding motor control circuit, but we do not propose a specific neural architecture for doing so here.

\section{DISCUSSION}

We have demonstrated that a neural field model can store previously visited locations in a search task with persistent activity. In a one-dimensional model, feedforward connectivity from a continuous attractor network that encodes position can initiate memory-encoding activity in the form of stationary fronts whose spatial resolution is determined by the frequency of underlying synaptic heterogeneity (as in Ref. [60]). Analysis of stationary solutions demonstrates the mechanism by which persistent activity expands in the memory layer is via hysteresis. For sufficiently strong input from the position layer, front positions in the vicinity of the position input undergo a bifurcation, leading to a rapid transition of the front to an adjacent stable location. We can capture the dynamics of these two layers by a low-dimensional approximation that tracks the interfaces of the front in the memory layer and the position of the bump attractor in the position layer. This low-dimensional model is leveraged to test the impact of memory-guided search. We find that search along a single one-dimensional segment

is not aided by memory-guided search, but search in more complex domains with distinct conjoined segments are. We expect that our approach can be extended to two-dimensional search processes, where memory-guided search is likely to be advantageous in most situations.

Our work contributes a new application of interface methods to neural field equations. Recently, the dynamics of labyrinthine and spot patterns in two dimensions have been captured by the low-dimensional projection of their interfaces $[61,69]$. This method has two advantages. First, it can lead to numerical simulation schemes that are an order of magnitude faster than simulating the full system, since the dimensionality of the dynamics can be reduced considerably. Second, it often leads to systems that are analytically tractable, allowing for a systematic study of both linear and nonlinear dynamics in the vicinity of equilibria. We leveraged both of these advantages in our work, since we were able to gain insight concerning the mechanism by which the memory layer dynamics evolved. Our work has shown that a searcher engaging memory from a neural field model storing previous positions can be approximately mapped to a ballistically searching agent with velocity evolving according to a memory-shaped jump process. This provides a new and interesting link between neural fields and low-dimensional models of stochastic search $[13,14]$.

Extensive experimental work has been performed to uncover the neural mechanisms underlying visual search. In particular, there is strong evidence that topographically organized ventral stream areas, such as V4, are involved in the memory and future planning of search saccades [34,38,40]. However, there have been few computational models that address the organization of neural circuits that would engender effective feedback between memory and search-planning brain regions. More evidence is also needed to confirm the importance of specific brain regions in oculomotor planning during visual search $[24,38]$. Our model suggests that reversible inactivation experiments may further shed light on which particular brain regions are responsible for memory-guided search. As was recently done to explore the functional significance of primate dorsal stream area for decision making [75], behavioral experiments in primates could be performed in the context of visual search, where candidate motor planning areas are temporarily inactivated. If the statistics of psychophysical performance are significantly different, this would suggest these areas play a role in the search guidance process. For instance, our model predicts that when an agent encounters a previously visited area in the visual field, the speed of their search should increase or even be directed away, related to recent results in Ref. [22].

Biophysical models of search tend to use memoryless agents, particularly because this can make for straightforward analysis and explicit results for quantities such as the mean first passage time to find the target [15]. However, there is evidence suggesting organisms employ memory of searched locations to find hidden targets in both foraging [7] and visual search [22] tasks. For this reason, we think it is worthwhile to develop techniques for understanding search models in the form of stochastic processes with various forms of memory. Our study is a first step in the direction of both implementing memory-driven stochastic search process in combination with a proposed neural mechanism for their implementation. 


\section{APPENDIX A: ONE-SIDED FRONT ANALYSIS}

Here we derive the condition for saddle-node (SN) bifurcations in the one-sided front version of stationary solutions to the full neural field model. As derived in the main text, we have a reduced system

$$
\begin{aligned}
& \theta_{u}=(b-a) e^{a-b}, \\
& \theta_{q}=\frac{1}{2}+\frac{\sigma \cos (n d)+n \sigma \sin (n d)}{2\left(n^{2}+1\right)}+\mathcal{P}(d ; a, b),
\end{aligned}
$$

for the interfaces of the bump in the position layer $(a, b)$, and the right interface of the front $d$. We can identify a curve of SN bifurcations in the reduced Eq. (10), corresponding to the critical values of $\left(I_{0}, d\right)$ that lead to wave propagation.

Fixing the threshold $\theta_{q}$, we can identify the critical $I_{0}$ for which a SN bifurcation occurs by simultaneously looking for the interface location $d^{c}$ and $I_{0}^{c}$ at which an extremum of the right-hand side of Eq. (10b) occurs. In essence, this identifies the point at which the bend of the bifurcation curves in Fig. 3 cross through a threshold value $\theta_{q}$ due to an increase of the input $I_{0}$. This requires, first, that Eq. (10b) is satisfied for $d=d^{c}$. Additionally, we require that the derivative of the right-hand side Eq. (10b) with respect to $d$ is zero since the SN bifurcation occurs at a critical point of the solution curve. This condition is given by the equation:

$$
\frac{n \sigma}{n^{2}+1}[n \cos (n d)-\sin (n d)]+\frac{\alpha I_{0}}{2}\left[e^{-\alpha|d-a|}-e^{-\alpha|d-b|}\right]=0,
$$

which can be solved explicitly for the critical input strength $I_{0}^{c}$ in terms of the interface location $d^{c}$ at the bifurcation:

$$
I_{0}^{c}=\frac{2 n \sigma}{\alpha\left(n^{2}+1\right)} \frac{\sin \left(n d^{c}\right)-n \cos \left(n d^{c}\right)}{e^{-\alpha\left|d^{c}-a\right|}-e^{-\alpha\left|d^{c}-b\right|}} .
$$

Plugging Eq. (A1) into Eq. (10b), we obtain the following implicit equation for the critical location of the interface, given the critical input $I_{0}^{c}$ :

$$
\begin{aligned}
\theta_{q}= & \frac{1}{2}+\sigma \frac{\cos \left(n d^{c}\right)+n \sin \left(n d^{c}\right)}{2\left(n^{2}+1\right)} \\
& +\frac{2 n \sigma}{\alpha\left(n^{2}+1\right)} \frac{\sin \left(n d^{c}\right)-n \cos \left(n d^{c}\right)}{e^{-\alpha\left|d^{c}-a\right|}-e^{-\alpha\left|d^{c}-b\right|}} \mathcal{P}\left(d^{c} ; a, b\right) .
\end{aligned}
$$

Equation (A2) further simplifies in the case where the input is ahead of the interface $d^{c}<a<b$, so that

$$
\begin{aligned}
\frac{2 \alpha}{\sigma}\left(n^{2}+1\right)\left(\theta_{q}-\frac{1}{2}\right)= & \left(\alpha-2 n^{2}\right) \cos \left(n d^{c}\right) \\
& +(\alpha+2) n \sin \left(n d^{c}\right),
\end{aligned}
$$

which can be solved explicitly,

$$
d^{c}=\frac{2}{n}\left[\tan ^{-1}\left(\frac{\sqrt{\mathcal{A}^{2}+\mathcal{B}^{2}-\mathcal{C}^{2}}+\mathcal{B}}{\mathcal{A}+\mathcal{C}}\right)+m \pi\right],
$$

for $m \in \mathbb{Z}$, where $\mathcal{A}=\alpha-2 n^{2}, \mathcal{B}=(\alpha+2) n$, and $\mathcal{C}=$ $\frac{2 \alpha}{\sigma}\left(n^{2}+1\right)\left(\theta_{q}-\frac{1}{2}\right)$. A similar set of explicit solutions can be obtained for the case $a<b<d^{c}$, so that

$$
d^{c}=\frac{2}{n}\left[\tan ^{-1}\left(\frac{\sqrt{\mathcal{A}^{2}+\mathcal{B}^{2}-\mathcal{C}^{2}}-\mathcal{B}}{\mathcal{C}-\mathcal{A}}\right)+m \pi\right] .
$$

We cannot solve the case $a<d^{c}<b$ explicitly, but it can easily be evaluated using numerical root finding.

\section{APPENDIX B: LINEAR STABILITY}

The stability of stationary solutions, defined by Eq. (9), can be determined by examining the evolution of spatiotemporal perturbations, $\epsilon[\psi(x, t), \phi(x, t)], 0<\epsilon \ll 1$, to the original stationary solutions $[U(x), Q(x)]$. We examine linearized equations associated with the perturbed solutions $u(x, t)=U(x)+\epsilon \psi(x, t)$ and $q(x, t)=Q(x)+\epsilon \phi(x, t)$. Plugging these into the original evolution Eqs. (1) and (2), for $v(t) \equiv 0$, and truncating to $\mathcal{O}(\epsilon)$, we obtain

$$
\begin{aligned}
& \psi_{t}=-\psi+w_{u} *\left[H^{\prime}\left(U-\theta_{u}\right) \psi\right], \\
& \phi_{t}=-\phi+w_{q} *\left[H^{\prime}\left(Q-\theta_{q}\right) \phi\right]+w_{p} *\left[H^{\prime}\left(U-\theta_{u}\right) \phi\right] .
\end{aligned}
$$

The spectrum of the associated linear operator is found by examining the evolution of the separable solutions $\psi(x, t)=$ $e^{\lambda t} \psi(x)$ and $\phi(x, t)=e^{\lambda t} \phi(x)$. Furthermore, the convolutions in Eq. (B1) are localized, since they involve derivatives of $H\left(U(x)-\theta_{u}\right)=H(x-a)-H(x-b)$ and $H(Q(x)-$ $\left.\theta_{q}\right)=H(x-c)-H(x-d)$, which are

$$
\begin{aligned}
& \delta(x-a)-\delta(x-b)=\frac{d H\left(U-\theta_{u}\right)}{d x}=H^{\prime}\left(U-\theta_{u}\right) U^{\prime}, \\
& \delta(x-c)-\delta(x-d)=\frac{d H\left(Q-\theta_{q}\right)}{d x}=H^{\prime}\left(Q-\theta_{q}\right) Q^{\prime},
\end{aligned}
$$

which can be rearranged to find

$$
\begin{aligned}
& H^{\prime}\left(U(x)-\theta_{u}\right)=\frac{1}{\left|U^{\prime}(a)\right|}[\delta(x-a)+\delta(x-b)], \\
& H^{\prime}\left(Q(x)-\theta_{q}\right)=\frac{\delta(x-c)}{\left|Q^{\prime}(c)\right|}+\frac{\delta(x-d)}{\left|Q^{\prime}(d)\right|},
\end{aligned}
$$

where

$$
\begin{aligned}
U^{\prime} & =w_{u}(x-a)-w_{u}(x-b), \\
Q^{\prime} & =\int_{c}^{d} \frac{d w_{q}(x, y)}{d x} d y+w_{p}(x-a)-w_{p}(x-b) .
\end{aligned}
$$

We can assume even symmetry of the bump solution $U(x)$, but not the front solution $Q(x)$. Applying the identities in Eq. (B2) to Eq. (B1) along with separability, we obtain the following system for the spectrum of the underlying linear operator:

$$
\begin{aligned}
(\lambda+1) \psi= & \gamma_{a}\left[w_{u}(x-a) \psi(a)+w_{u}(x-b) \psi(b)\right], \\
(\lambda+1) \phi= & \gamma_{a}\left[w_{p}(x-a) \psi(a)+w_{p}(x-b) \psi(b)\right] \\
& +\gamma_{c} w_{q}(x, c) \phi(c)+\gamma_{d} w_{q}(x, d) \phi(d),
\end{aligned}
$$

where $\gamma_{a}^{-1}=\left|U^{\prime}(a)\right|, \quad \gamma_{c}^{-1}=\left|Q^{\prime}(c)\right|$, and $\gamma_{d}^{-1}=\left|Q^{\prime}(d)\right|$. There are two classes of solution to Eq. (B3). First, all solutions $\psi(a)=\psi(b)=\phi(c)=\phi(d)=0$ lie in the essential spectrum and $\lambda=-1$, which contributes to no instabilities. Solutions that do not satisfy the condition $\psi(a)=$ $\psi(b)=\phi(c)=\phi(d)=0$ can be classified by the vector $[\psi(a), \psi(b), \phi(c), \phi(d)]$. In this case, the functions $[\psi(x), \phi(x)]$ are fully specified by their values at $(a, b, c, d)$. This leads to 
the linear system

$$
\begin{aligned}
(\lambda+1) \psi(a)= & \gamma_{a}\left[w_{u}(0) \psi(a)+w_{u}(b-a) \psi(b)\right], \\
(\lambda+1) \psi(b)= & \gamma_{a}\left[w_{u}(b-a) \psi(a)+w_{u}(0) \psi(b)\right], \\
(\lambda+1) \phi(c)= & \gamma_{a}\left[w_{p}(c-a) \psi(a)+w_{p}(c-b) \psi(b)\right] \\
& +\gamma_{c} w_{q}(c, c) \phi(c)+\gamma_{d} w_{q}(c, d) \phi(d), \\
(\lambda+1) \phi(d)= & \gamma_{a}\left[w_{p}(d-a) \psi(a)+w_{p}(d-b) \psi(b)\right] \\
& +\gamma_{c} w_{q}(d, c) \phi(c)+\gamma_{d} w_{q}(d, d) \phi(d) .
\end{aligned}
$$

Since Eq. (B4) is in block triangular form, the eigenvalue problem can be separated in to diagonal blocks [76]. The upper left block yields a two-by-two eigenvalue problem with eigenvectors $[\psi(a), \psi(b)][48,53,77]$ :

$$
\begin{aligned}
& (\lambda+1) \psi(a)=\gamma_{a}\left[w_{u}(0) \psi(a)+w_{u}(b-a) \psi(b)\right], \\
& (\lambda+1) \psi(b)=\gamma_{a}\left[w_{u}(b-a) \psi(a)+w_{u}(0) \psi(b)\right],
\end{aligned}
$$

leading to the following characteristic equations for the associated eigenvalues:

$$
\begin{aligned}
\mathcal{U}(\lambda) & =\left|\begin{array}{cc}
\lambda+1-\gamma_{a} w_{u}(0) & -\gamma_{a} w_{u}(b-a) \\
-\gamma_{a} w_{u}(b-a) & \lambda+1-\gamma_{a} w_{u}(0)
\end{array}\right| \\
& =\lambda\left[\lambda-\frac{2 w_{u}(b-a)}{w_{u}(0)-w_{u}(b-a)}\right]=0 .
\end{aligned}
$$

The other two-by-two system corresponds to eigenvectors of the form $[\phi(c), \phi(d)]$ :

$$
\begin{aligned}
& (\lambda+1) \phi(c)=\gamma_{c} w_{q}(c, c) \phi(c)+\gamma_{d} w_{q}(c, d) \phi(d), \\
& (\lambda+1) \phi(d)=\gamma_{c} w_{q}(d, c) \phi(c)+\gamma_{d} w_{q}(d, d) \phi(d),
\end{aligned}
$$

with corresponding characteristic equation

$$
\begin{aligned}
\mathcal{Q}(\lambda) & =\left|\begin{array}{cc}
\lambda+1-\gamma_{c} w_{q}(c, c) & -\gamma_{d} w_{q}(c, d) \\
-\gamma_{c} w_{q}(d, c) & \lambda+1-\gamma_{d} w_{q}(d, d)
\end{array}\right| \\
& =\lambda^{2}+\mathcal{Q}_{1} \lambda+\mathcal{Q}_{0}=0,
\end{aligned}
$$

where $\mathcal{Q}_{0}=\left[\gamma_{c} w_{q}(c, c)-1\right]\left[\gamma_{d} w_{q}(d, d)-1\right]-\gamma_{c} \gamma_{d} w_{q}(c, d)$ $w_{q}(d, c)$ and $\mathcal{Q}_{1}=2-\gamma_{c} w_{q}(c, c)-\gamma_{d} w_{q}(d, d)$. Clearly, the roots of $\mathcal{U}(\lambda)=0$ are $\lambda_{0}=0$ and $\lambda_{w}=2 w_{u}(b-a) /\left[w_{u}(0)-\right.$ $\left.w_{u}(b-a)\right]$, the typical stability classification of bumps in neural fields with Heaviside firing rates [48,53]. The zero eigenvalue indicates the translation symmetry of the bump, and the generically nonzero eigenvalue $\lambda_{w}$ represents the stability of the bump in response to width perturbations, determined by the sign of $w_{u}(b-a)$. We are interested in the linear stability characterized by $\mathcal{Q}(\lambda)=0$. These eigenvalues can be determined explicitly assuming $(a, b, c, d)$ and $Q^{\prime}(x)$ are known by applying the quadratic formula

$$
\lambda_{ \pm}=\frac{1}{2}\left[-\mathcal{Q}_{1}-\sqrt{\mathcal{Q}_{1}^{2}-4 \mathcal{Q}_{0}}\right] .
$$

Neutral stability of the front occurs when $\operatorname{Re} \lambda_{+}=0$. Past work showed these are SN bifurcations [60], so we expect $\lambda_{+}=0$. Placing this condition on Eq. (B6) yields $\mathcal{Q}_{0}=0$, and

$$
\left[\gamma_{c} w_{q}(c, c)-1\right]\left[\gamma_{d} w_{q}(d, d)-1\right]=\gamma_{c} \gamma_{d} w_{q}(c, d) w_{q}(d, c) .
$$

For front interfaces that are far apart $d-c \gg 1$, $\left|w_{q}(c, d)\right|,\left|w_{q}(d, c)\right| \ll 1$. These terms scale exponentially with the distance $d-c$, so their product will be smaller, and we approximate Eq. (B5) as a diagonal system with eigenvalues $\lambda_{d}:=\lambda_{+}=\gamma_{d} w_{q}(d, d)-1$ and $\lambda_{c}:=\lambda_{-}=\gamma_{c} w_{q}(c, c)-1$. Focusing on bifurcations that emerge at the right interface near $x=d$, we expect the SN bifurcation to occur when $\lambda_{d}=0$ or $w_{q}(d, d)=\left|Q^{\prime}(d)\right|$. This is identical to the condition we derived above for the location of SN bifurcations for a single-interface front.

The associated eigenfunction is determined by plugging $\lambda_{d}=\lambda_{+}$into Eq. (B4), solving the linear system for the degenerate eigenvector $[\psi(a), \psi(b), \phi(c), \phi(d)]$, and using the full linear system Eq. (B3) to determine the shape of $[\psi(x), \phi(x)]$.

\section{APPENDIX C: DERIVING THE INTERFACE EQUATIONS}

To start, we define the active regions of both layers, $A_{u}(t)=$ $\left\{x \mid u(x, t)>\theta_{u}\right\}$ and $A_{q}(t)=\left\{x \mid q(x, t)>\theta_{q}\right\}$ where the output of the firing rate nonlinearities will be nonzero, allowing us to rewrite Eqs. (1) and (2) as

$$
\begin{aligned}
& u_{t}+u=\int_{A_{u}(t)} w_{u}(x-y) d y-v(t) \int_{A_{u}(t)} w_{u}^{\prime}(x-y) d y, \\
& q_{t}+q=\int_{A_{q}(t)} w_{q}(x, y) d y+\int_{A_{u}(t)} w_{p}(x-y) d y,
\end{aligned}
$$

and since we expect the active regions to be simply connected, we specify

$$
A_{u}(t)=\left[x_{-}(t), x_{+}(t)\right], \quad A_{q}(t)=\left[\Delta_{-}(t), \Delta_{+}(t)\right] .
$$

Assuming continuity of $u(x, t)$ and $q(x, t)$, the boundaries of $A_{u, q}(t)$ describe the interfaces of the bump and front. Thus, we write the dynamic threshold equations

$$
u\left(x_{ \pm}(t), t\right)=\theta_{u}, \quad q\left(\Delta_{ \pm}(t), t\right)=\theta_{q} .
$$

Differentiating Eq. (C2) with respect to $t$, we find

$$
\begin{aligned}
\alpha_{ \pm}(t) \frac{d x_{ \pm}}{d t}+\frac{\partial u\left(x_{ \pm}(t), t\right)}{\partial t} & =0, \\
\beta_{ \pm}(t) \frac{d \Delta_{ \pm}}{d t}+\frac{\partial q\left(\Delta_{ \pm}(t), t\right)}{\partial t} & =0
\end{aligned}
$$

where we have defined

$$
\alpha_{ \pm}(t)=\frac{\partial u\left(x_{ \pm}(t), t\right)}{\partial x}, \quad \beta_{ \pm}(t)=\frac{\partial q\left(\Delta_{ \pm}(t), t\right)}{\partial x} .
$$

We obtain differential equations for the dynamics of the interfaces $x_{ \pm}(t)$ and $\Delta_{ \pm}(t)$ by rearranging Eq. (C3) and substituting Eq. (C1) in to yield

$$
\begin{aligned}
\frac{d x_{ \pm}}{d t}= & -\frac{1}{\alpha_{ \pm}(t)}\left[\int_{x_{-}(t)}^{x_{+}(t)} w_{u}\left(x_{ \pm}(t)-y\right) d y\right. \\
& \left. \pm v(t)\left(w_{u}(0)-w_{u}\left(x_{+}(t)-x_{-}(t)\right)\right)-\theta_{u}\right], \\
\frac{d \Delta_{ \pm}}{d t}= & -\frac{1}{\beta_{ \pm}(t)}\left[\int_{\Delta_{-}(t)}^{\Delta_{+}(t)} w_{q}\left(\Delta_{ \pm}(t), y\right) d y\right. \\
& \left.+\int_{x_{-}(t)}^{x_{+}(t)} w_{p}\left(\Delta_{ \pm}(t)-y\right) d y-\theta_{q}\right] .
\end{aligned}
$$


To further simplify Eq. (C4), we assume $x_{+}(t)-x_{-}(t) \approx$ $b-a$, based on our linear stability analysis showing bumps are stable to width perturbations, but marginally stable to position perturbations. This is means we assume $u(x, t) \approx$ $U\left(x-\Delta_{u}(t)\right)$, so the neural activity variable is roughly a temporal translation of a stationary bump [78]. In this case, the first integral term in Eq. (C4) and $\theta_{u}$ cancel. Thus, $w_{u}(0)-w_{u}\left(x_{+}(t)-x_{-}(t)\right) \approx\left|U^{\prime}(a)\right|$. We also approximate the spatial gradients using the stationary solutions, $\alpha_{-}(t) \approx U^{\prime}(a), \alpha_{+}(t) \approx U^{\prime}(b), \beta_{-}(t) \approx Q^{\prime}(c)$, and $\beta_{+}(t)=$ $Q^{\prime}(d)$. Such an approximation will hold in the limit of small changes to the spatial gradient of the bump and front interfaces, $u(x, t)=U(x)+\varepsilon \Psi(x, t)+\mathcal{O}\left(\varepsilon^{2}\right)$ and $q(x, t)=$ $Q(x)+\varepsilon \Phi(x, t)+\mathcal{O}\left(\varepsilon^{2}\right)$, since then $u_{x}(x, t)=U^{\prime}(x)+\mathcal{O}(\varepsilon)$ and $q_{x}(x, t)=Q^{\prime}(x)+\mathcal{O}(\varepsilon)$. Since the terms inside the brackets of Eq. (C4b) are $\mathcal{O}(\varepsilon)$, performing an $\mathcal{O}(1)$ approximation of $u_{x}$ and $q_{x}$ constitutes a linear approximation of the dynamics of $\Delta_{ \pm}(t)$. This combination of approximations leads to a simplified set of interface equations. The edges of the bump propagate according to the velocity input

$$
x_{ \pm}(t)=\int_{0}^{t} v(s) d s+x_{ \pm}(0)
$$

so we assign $\Delta_{u}(t)=\left[x_{+}(t)+x_{-}(t)\right] / 2$ to be the location of the bump in the position layer, and

$$
\Delta_{u}(t)=\int_{0}^{t} v(s) d s+\Delta_{u}(0),
$$

where assuming $x_{+}(t)-x_{-}(t) \approx b-a=2 h, \quad x_{ \pm}(t)=$ $\Delta_{u}(t) \pm h$. The front interface equations are then approximated with the stationary gradient assumptions discussed above:

$$
\begin{aligned}
\frac{d \Delta_{+}}{d t}= & \gamma_{d}\left[\int_{\Delta_{-}(t)}^{\Delta_{+}(t)} w_{q}\left(\Delta_{+}(t), y\right) d y\right. \\
& \left.+\int_{\Delta_{+}(t)-\Delta_{u}(t)-h}^{\Delta_{+}(t)-\Delta_{u}(t)+h} w_{p}(y) d y-\theta_{q}\right], \\
\frac{d \Delta_{-}}{d t}= & -\gamma_{c}\left[\int_{\Delta_{-}(t)}^{\Delta_{+}(t)} w_{q}\left(\Delta_{-}(t), y\right) d y\right. \\
& \left.+\int_{\Delta_{-}(t)-\Delta_{u}(t)-h}^{\Delta_{-}(t)-\Delta_{u}(t)+h} w_{p}(y) d y-\theta_{q}\right],
\end{aligned}
$$

where $\gamma_{c}^{-1}=\left|Q^{\prime}(c)\right|$ and $\gamma_{d}^{-1}=\left|Q^{\prime}(d)\right|$, so $\Delta_{u}(t)$ integrates the weak velocity input, and the front interfaces $\Delta_{ \pm}(t)$ interact nonlinearly with the location of the bump.

To simplify Eq. (C6) further, we compute integrals, recalling $w_{q}(x, y)$ and $w_{p}(x)$ are given by Eqs. (4) and (5). The corresponding integrals are essentially the same as those evaluated in Eqs. (6) and (8). The resulting formulas simplify, since we are only examining the dynamics along the interfaces:

$$
\begin{aligned}
\frac{d \Delta_{+}}{d t}= & \gamma_{d}\left[\sigma \frac{\cos \left[n \Delta_{+}(t)\right]+n \sin \left[n \Delta_{+}(t)\right]}{2\left(n^{2}+1\right)}\right. \\
& \left.+I_{0} G\left(\Delta_{+}(t)-\Delta_{u}(t)\right)+\frac{1}{2}-\theta_{q}\right], \\
\frac{d \Delta_{-}}{d t}= & -\gamma_{c}\left[\sigma \frac{\cos \left(n \Delta_{-}(t)\right)-n \sin \left(n \Delta_{-}(t)\right)}{2\left(n^{2}+1\right)}\right. \\
& \left.+I_{0} G\left(\Delta_{-}(t)-\Delta_{u}(t)\right)+\frac{1}{2}-\theta_{q}\right],
\end{aligned}
$$

where $G(\Delta)=\mathcal{S}(h-\Delta)+\mathcal{S}(\Delta+h)$ and $\mathcal{S}(x)=\operatorname{sgn}(x)$ $\left(1-e^{-\alpha|x|}\right)$, and $e^{-\left|\Delta_{+}-\Delta_{-}\right|} \approx 0$, assuming large interface separation. Assuming $\Delta_{+}-\Delta_{-} \gg 1$ also allows us to approximate the spatial gradients $\gamma_{c}=\gamma_{d}$, so it suffices to use Eq. (10b) in the weak input, $I_{0} \ll 1$, limit, yielding

$$
d=\frac{2}{n} \tan ^{-1}\left(\frac{\sqrt{\sigma^{2}+n^{2} \sigma^{2}-\left(2 \theta_{q}-1\right)^{2}\left(n^{2}+1\right)^{2}}+n \sigma}{\sigma+\left(2 \theta_{q}-1\right)\left(n^{2}+1\right)}\right),
$$

up to periodicity, so that

$$
\gamma_{c}^{-1}=\gamma_{d}^{-1}=-Q^{\prime}(d)=\frac{1}{2}\left[1+\sigma \frac{\cos (n d)+n \sin (n d)}{n^{2}+1}\right] .
$$

\section{APPENDIX D: BALLISTIC SEARCHER MODEL}

\section{Approximating interface model with control}

We map the activities of the position $u(x, t)$ and memory $(q(x, t))$ layers directly onto a velocity variable:

$$
v(t)=\chi[u(x, t), q(x, t)] \cdot \bar{v}(t),
$$

so that $\bar{v}(t)$ is an open-loop component of the velocity control, not subject to the internal neural activity variables. The openloop component is then modulated by a closed-loop control $\chi(u, q)$ with evolution equation

$$
\begin{aligned}
\tau_{\chi} \dot{\chi}(t)= & 2\left\langle H\left(u-\theta_{u}\right), H\left(q-\theta_{q}\right)\right\rangle\left[\left(\chi_{+}-\chi(t)\right]\right. \\
& -\left\langle H\left(u-\theta_{u}\right), 1\right\rangle\left[\chi_{-}-\chi(t)\right],
\end{aligned}
$$

where $\langle F, G\rangle=\int_{-\infty}^{\infty} F(x) G(x) d x$, so when the position layer totally overlaps with the memory layer, both inner products equal $\Delta_{u}(t)$, the width of the bump. The steady state control input in this case of total overlap is $\chi(t) \rightarrow 2 \chi_{+}-\chi_{-}$, whereas when the position layer does not overlap with the memory layer, $\chi(t) \rightarrow \chi_{-}$. The parameters $\chi_{ \pm}$are fixed and thus determine the maximal and minimal possible value of the control feedback function $\chi(t)$. Search will speed up (slow down) when the agent is in a location it has already visited for $\chi_{+}>\chi_{-}\left(\chi_{-} / 2<\chi_{+}<\chi_{-}\right)$. In this way, memory of previous positions can guide search.

Now, to analyze the impact of this memory-guided search strategy, we consider the limit of the interface Eq. (11) in which a searcher starts in the left side of a bounded domain. All our calculations to this point assumed $x \in(-\infty, \infty)$, an approximation to the case of large bounded domains of length $L \gg 1$ we analyze now. In this case, the left interface $\Delta_{-}(t)$ is irrelevant. Furthermore, we study the limit in which the position input dominates the dynamics. Taking $\theta_{q} \rightarrow 1 / 2$, $\sigma \rightarrow 0$ and assuming a velocity with constant amplitude to start $|v(t)|=v_{0}$, we then have

$$
\frac{d \Delta_{+}}{d t}=\gamma_{d} I_{0} G\left(\Delta_{+}(t)-v_{0} t\right)
$$

by plugging into Eq. (11), where we have $\left|\Delta_{u}(t)\right|=v_{0} t$. Switches in the direction of the velocity occur when the agent encounters the boundary of the domain, $v(t) \mapsto-v(t)$. The agent begins moving rightward: $v(t)=v_{0}>0$. The corresponding phase shift between the front interface position and the bump position is $\Theta=v_{0} t-\Delta_{+}(t)$, so $\Delta_{+}(t)=v_{0} t-\Theta$. We thus expect constant velocity solutions for $\Delta_{+}(t)$ as long 
as the condition

$$
v_{0}=\gamma_{d} I_{0} G(-\Theta)
$$

holds for some phase shift $\Theta$. Note also since we are using closed loop control, this solution will only be valid if $v_{0}$ is the constant velocity to which the control loop has equilibrated. Self-consistency of Eqs. (D1) and (D2) then requires $v_{0}=$ $\bar{\chi}_{0} \cdot \bar{v}$, where

$$
\bar{\chi}_{0}=\frac{2 \max (h-\Theta, 0) \chi_{+}-2 h \chi_{-}}{2 \max (h-\Theta, 0)-2 h},
$$

where $h$ is the half-width of the bump in the position layer. Once the domain has been searched, the control variable is updated to $\bar{\chi}_{1}=2 \chi_{+}-\chi_{-}$, and we thus define $v_{1}=\bar{\chi}_{1} \cdot \bar{v}$. Since the parameters $\chi_{ \pm}$can be tuned to give any pair $\left(v_{0}, v_{1}\right)$, we focus on the limit $\tau_{\chi} \rightarrow 0$, and assume the agent employs these two search velocities, depending on a novel or searched regime.

\section{Single-segment mean first passage time}

Here we present the calculation of the generalized mean first passage time for an agent searching along a single segment. The first visit to the target occurs after $T_{L}\left(v_{0}\right)=\left(x_{T}-r\right) / v_{0}$. During the first pass over the target, the searcher discovers the target with probability $P_{v_{0}}$, Eq. (12), with conditional mean time within the target $T_{a}\left(v_{0}\right)$, Eq. (13). The time between the first and the second visits is $T_{R}\left(v_{0}\right)+T_{R}\left(v_{1}\right)$, where $T_{R}(v)=$ $\left(L-x_{T}-r\right) / v$, and the probability of finding the trap during the next visit is $P_{v_{1}}$ with mean time $T_{a}\left(v_{1}\right)$. Subsequent times and probabilities are computed similarly, and the time spent searching scales linearly with the length of the searcher's path. Using geometric series, we compute the mean time to find the target by marginalizing over all possible visit counts

$$
\begin{aligned}
T\left(x_{T}\right)= & T_{L}\left(v_{0}\right)+P_{v_{0}} T_{a}\left(v_{0}\right)+\frac{1-P_{v_{0}}}{2-P_{v_{1}}}\left[\frac{2 L}{v_{1} P_{v_{1}}}\right. \\
& +\left(1-P_{v_{1}}\right)\left(T_{v_{0}}+T_{R}\left(v_{0}\right)+T_{L}\left(v_{1}\right)+T_{a}\left(v_{1}\right)-\frac{L}{v_{1}}\right) \\
& \left.+T_{v_{0}}+T_{R}\left(v_{0}\right)+T_{R}\left(v_{1}\right)+T_{a}\left(v_{1}\right)-\frac{2 L}{v_{1}}\right] .
\end{aligned}
$$

The generalized mean first passage time is given by integrating over the range of possible target locations $x_{T}$, assuming a uniform probability of placement: $\bar{T}=$ $\frac{1}{L-2 r} \int_{r}^{L-r} T\left(x_{T}\right) d x_{T}$. Since the only terms in Eq. (D3) that depend on $x_{T}$ are $T_{L}(v)$ and $T_{R}(v)$, we need only compute $\bar{T}_{L}(v)=\frac{1}{L-2 r} \int_{r}^{L-r} \frac{x_{T}-r}{v} d x_{T}=\frac{L-2 r}{2 v}$ and $\bar{T}_{R}(v)=$ $\frac{1}{L-2 r} \int_{r}^{L-r} \frac{L-x_{T}-r}{v} d x_{T}=\frac{L-2 r}{2 v}$, and we rescale space, so it is in units of the radius $r$. This is equivalent to setting $r=1$ in Eq. (D3), and considering spatial parameters in units of $r$, which yields

$$
\begin{aligned}
\bar{T}= & \frac{L-2}{2 v_{0}}+P_{v_{0}} T_{a}\left(v_{0}\right)+\left(1-P_{v_{0}}\right)\left[\frac{L}{v_{1} P_{v_{1}}}\right. \\
& \left.+\left(1+\frac{L}{2}\right)\left(\frac{1}{v_{0}}-\frac{1}{v_{1}}\right)+T_{a}\left(v_{1}\right)\right] .
\end{aligned}
$$

[1] T. T. Hills, P. M. Todd, D. Lazer, A. D. Redish, I. D. Couzin, and C. S. R. Group, Trends Cognit. Sci. 19, 46 (2015).

[2] T. Mueller and W. F. Fagan, Oikos 117, 654 (2008).

[3] I. Kanitscheider and I. Fiete, Curr. Opin. Syst. Biol. 3, 186 (2017).

[4] N. D. Daw, J. P. O’Doherty, P. Dayan, B. Seymour, and R. J. Dolan, Nature (London) 441, 876 (2006).

[5] J. D. Cohen, S. M. McClure, and J. Y. Angela, Philos. Trans. R. Soc. London, Ser. B 362, 933 (2007).

[6] K. Mehlhorn, B. R. Newell, P. M. Todd, M. D. Lee, K. Morgan, V. A. Braithwaite, D. Hausmann, K. Fiedler, and C. Gonzalez, Decision 2, 191 (2015).

[7] S. J. Simpson, R. M. Sibly, K. P. Lee, S. T. Behmer, and D. Raubenheimer, Anim. Behav. 68, 1299 (2004).

[8] T. T. Hills, M. N. Jones, and P. M. Todd, Psychol. Rev. 119, 431 (2012).

[9] E. Charnov and G. H. Orians, Optimal Foraging: Some Theoretical Explorations (UNM Digital Repository, Albuquerque, New Mexico, 2006).

[10] F. Bartumeus and J. Catalan, J. Phys. A: Math. Theor. 42, 434002 (2009).

[11] O. Bénichou, C. Loverdo, M. Moreau, and R. Voituriez, Rev. Mod. Phys. 83, 81 (2011).

[12] S. S. Andrews and D. Bray, Phys. Biol. 1, 137 (2004).

[13] O. Bénichou, M. Coppey, M. Moreau, P. H. Suet, and R. Voituriez, Phys. Rev. Lett. 94, 198101 (2005).

[14] J. M. Newby and P. C. Bressloff, Bull. Math. Biol. 72, 1840 (2010).
[15] G. M. Viswanathan, V. Afanasyev, S. V. Buldyrev, E. J. Murphy, P. A. Prince, and H. E. Stanley, Nature (London) 381, 413 (1996).

[16] T. L. Thornton and D. L. Gilden, Psychol. Rev. 114, 71 (2007).

[17] M. I. Posner and Y. Cohen, Components of Visual Orienting, Attention and performance $\mathrm{X}$ : Control of language processes, Vol. 32 (1984), pp. 531-556.

[18] R. Klein, Nature (London) 334, 430 (1988).

[19] T. S. Horowitz and J. M. Wolfe, Nature (London) 394, 575 (1998).

[20] Z. Wang and R. M. Klein, Vision Res. 50, 220 (2010).

[21] T. J. Smith and J. M. Henderson, J. Vision 11, 3 (2011).

[22] P. M. Bays and M. Husain, J. Vision 12, 8 (2012).

[23] A. R. Mayer, M. Seidenberg, J. M. Dorflinger, and S. M. Rao, J. Cognitive Neurosci. 16, 1262 (2004).

[24] G. D. Horwitz and W. T. Newsome, Science 284, 1158 (1999).

[25] A. Sapir, N. Soroker, A. Berger, and A. Henik, Nat. Neurosci. 2, 1053 (1999).

[26] J. Lepsien and S. Pollmann, J. Cognitive Neurosci. 14, 127 (2002).

[27] T. Ro, A. Farnè, and E. Chang, Exp. Brain Res. 150, 290 (2003).

[28] C. E. Curtis and M. D'Esposito, Trends Cognit. Sci. 7, 415 (2003).

[29] S.-H. Oh and M.-S. Kim, Psychon. Bull. Rev. 11, 275 (2004).

[30] H. Melville, Bartleby, the Scrivener (State University of New York, Albany, 1999).

[31] http://www.garystpc.com/pest-id/gophers-moles.php

[32] M. Handford, Where's Waldo? (Little, Brown, Boston, 1987).

[33] http://www.cs.colorado.edu/department/maps/ec.html. 
[34] L. Chelazzi, E. K. Miller, J. Duncan, and R. Desimone, Nature (London) 363, 345 (1993).

[35] L. Chelazzi, J. Duncan, E. K. Miller, and R. Desimone, J. Neurophysiol. 80, 2918 (1998).

[36] R. Desimone, Proc. Nat. Acad. Sci. USA 93, 13494 (1996).

[37] S. J. Luck, L. Chelazzi, S. A. Hillyard, and R. Desimone, J. Neurophysiol. 77, 24 (1997).

[38] J. A. Mazer and J. L. Gallant, Neuron 40, 1241 (2003).

[39] B. J. White, D. J. Berg, J. Y. Kan, R. A. Marino, L. Itti, and D. P. Munoz, Nat. Commun. 8, 14263 (2017).

[40] N. P. Bichot, A. F. Rossi, and R. Desimone, Science 308, 529 (2005).

[41] T. K. Sato, I. Nauhaus, and M. Carandini, Neuron 75, 218 (2012).

[42] L. Muller, A. Reynaud, F. Chavane, and A. Destexhe, Nat. Commun. 5, 3675 (2014).

[43] T. P. Zanos, P. J. Mineault, K. T. Nasiotis, D. Guitton, and C. C. Pack, Neuron 85, 615 (2015).

[44] E. Aksay, G. Gamkrelidze, H. Seung, R. Baker, and D. Tank, Nat. Neurosci. 4, 184 (2001).

[45] B. L. McNaughton, F. P. Battaglia, O. Jensen, E. I. Moser, and M.-B. Moser, Nat. Rev. Neurosci. 7, 663 (2006).

[46] Y. Burak and I. R. Fiete, PLoS Comput. Biol. 5, e1000291 (2009).

[47] R. Engbert, K. Mergenthaler, P. Sinn, and A. Pikovsky, Proc. Nat. Acad. Sci. USA 108, E765 (2011).

[48] S. Amari, Biol. Cybern. 27, 77 (1977).

[49] H. S. Seung, Proc. Nat. Acad. Sci. USA 93, 13339 (1996).

[50] K. Zhang, J. Neurosci. 16, 2112 (1996).

[51] S. Coombes and M. R. Owen, Phys. Rev. Lett. 94, 148102 (2005).

[52] Z. P. Kilpatrick and P. C. Bressloff, Physica D (Amsterdam, Neth.) 239, 1048 (2010).

[53] P. C. Bressloff, J. Phys. A: Math. Theor. 45, 033001 (2012).

[54] S. Coombes and H. Schmidt, Discr. Contin. Dyn. Syst., Ser. A. 28, 1369 (2010).

[55] T. Horowitz and J. Wolfe, Visual Cognition 10, 257 (2003).

[56] M. Stemmler, M. Usher, and E. Niebur, Science 269, 1877 (1995).

[57] A. Angelucci and P. C. Bressloff, Prog. Brain Res. 154, 93 (2006).
[58] P. C. Bressloff, Physica D (Amsterdam, Neth.) 155, 83 (2001).

[59] S. Coombes and C. R. Laing, Phys. Rev. E 83, 011912 (2011).

[60] D. Avitabile and H. Schmidt, Physica D (Amsterdam, Neth.) 294, 24 (2015).

[61] S. Coombes, H. Schmidt, and I. Bojak, J. Math. Neurosci. 2, 9 (2012).

[62] J. M. Wolfe, E. M. Palmer, and T. S. Horowitz, Vision Res. 50, 1304 (2010).

[63] D. S. Olton, C. Collison, and M. A. Werz, Learning Motivation 8, 289 (1977).

[64] S. B. Floresco, J. K. Seamans, and A. G. Phillips, J. Neurosci. 17, 1880 (1997).

[65] D. B. Poll and Z. P. Kilpatrick, J. Stat. Mech.: Theory Exp. (2016) 053201.

[66] C. R. Laing and W. C. Troy, SIAM J. Appl. Dyn. Syst. 2, 487 (2003).

[67] S. E. Folias and P. C. Bressloff, SIAM J. Appl. Dyn. Syst. 3, 378 (2004).

[68] M. Owen, C. Laing, and S. Coombes, New J. Phys. 9, 378 (2007).

[69] A. Gökçe, D. Avitabile, and S. Coombes, J. Math. Neurosci. 7, 12 (2017).

[70] D. Poll and Z. P. Kilpatrick, SIAM J. Appl. Math. 75, 1553 (2015).

[71] S. Deneve, P. E. Latham, and A. Pouget, Nat. Neurosci. 2, 740 (1999).

[72] S. R. Olsen, V. Bhandawat, and R. I. Wilson, Neuron 66, 287 (2010).

[73] J. M. Beck, P. E. Latham, and A. Pouget, J. Neurosci. 31, 15310 (2011).

[74] M. M. Churchland, J. P. Cunningham, M. T. Kaufman, J. D. Foster, P. Nuyujukian, S. I. Ryu, and K. V. Shenoy, Nature (London) 487, 51 (2012).

[75] L. N. Katz, J. L. Yates, J. W. Pillow, and A. C. Huk, Nature (London) 535, 285 (2016).

[76] J. R. Silvester, Math. Gazette 84, 460 (2000).

[77] B. Ermentrout, Rep. Prog. Phys. 61, 353 (1998).

[78] Z. P. Kilpatrick and B. Ermentrout, SIAM J. Appl. Dyn. Syst. 12, 61 (2013). 